Article

\title{
Karyotype Diversity, Mode, and Tempo of the Chromosomal Evolution of Attina (Formicidae: Myrmicinae: Attini): Is There an Upper Limit to Chromosome Number?
}

\author{
Danon Clemes Cardoso*(D) and Maykon Passos Cristiano \\ Departamento de Biodiversidade, Evolução e Meio Ambiente, Universidade Federal de Ouro Preto, \\ Ouro Preto 35400-000, Brazil; maykon@ufop.edu.br \\ * Correspondence: danon@ufop.edu.br; Tel.: +55-3135591214
}

check for updates

Citation: Cardoso, D.C.; Cristiano, M.P. Karyotype Diversity,

Mode, and Tempo of the Chromosomal Evolution of Attina (Formicidae: Myrmicinae: Attini): Is There an Upper Limit to Chromosome Number? Insects 2021, 12, 1084. https:// doi.org/10.3390/insects12121084

Academic Editor: Valentina G. Kuznetsova

Received: 7 October 2021

Accepted: 30 November 2021

Published: 2 December 2021

Publisher's Note: MDPI stays neutral with regard to jurisdictional claims in published maps and institutional affiliations.

Copyright: (c) 2021 by the authors. Licensee MDPI, Basel, Switzerland. This article is an open access article distributed under the terms and conditions of the Creative Commons Attribution (CC BY) license (https:// creativecommons.org/licenses/by/ $4.0 /)$.
Simple Summary: Ants are an important insect group that includes a considerable number of species. Along with this diversity in species, they also exhibit a wide variation in chromosome numbers, from 1 up to 60 chromosomes. DNA molecules can be counted in a specific stage of the cell life cycle and quantified. These DNA molecules are very tightly packed together with several proteins and are called chromosomes. Each species shows a specific number of chromosomes with different shapes and sizes, as well as different quantities of DNA. We can use such information (the number of chromosomes, shape of the chromosomes, and quantity of DNA) as morphological attributes to study evolution at the species level. In this study, we describe new karyotypes of several ant species. In addition, from previous studies, we have compiled all the available information regarding the chromosome number and DNA quantity in fungus-farming ant cells. Different processes, called rearrangements, can change chromosomes over time, producing new character states. Such states can be tracked, along with the species and groups of similar species, using their relationships to identify patterns. We use DNA sequences to reconstruct the relationships of fungus-farming ant species (molecular phylogeny). By comparing such phylogeny with the chromosome number and DNA quantity, we discuss the evolution of chromosomes and DNA quantity (or genome size), and the potential limits to these features across fungus-farming ants.

Abstract: Ants are an important insect group that exhibits considerable diversity in chromosome numbers. Some species show only one chromosome, as in the males of the Australian bulldog ant Myrmecia croslandi, while some have as many as 60 chromosomes, as in the males of the giant Neotropical ant Dinoponera lucida. Fungus-growing ants are a diverse group in the Neotropical ant fauna, engaged in a symbiotic relationship with a basidiomycete fungus, and are widely distributed from Nearctic to Neotropical regions. Despite their importance, new chromosome counts are scarcely reported, and the marked variation in chromosome number across species has been poorly studied under phylogenetic and genome evolutionary contexts. Here, we present the results of the cytogenetic examination of fungus-farming ants and compile the cytogenetic characteristics and genome size of the species studied to date to draw insights regarding the evolutionary paths of karyotype changes and diversity. These data are coupled with a fossil-calibrated phylogenetic tree to discuss the mode and tempo of chromosomal shifting, considering whether there is an upper limit for chromosome number and genome size in ants, using fungus-farming ants as a model study. We recognize that karyotypes are generally quite variable across fungus-farming ant phylogeny, mostly between genera, and are more numerically conservative within genera. A low chromosome number, between 10 and 12 chromosomes, seems to present a notable long-term evolutionary stasis (intermediate evolutionary stasis) in fungus-farming ants. All the genome size values were inside a limited spectrum below $1 \mathrm{pg}$. Eventual departures in genome size occurred with regard to the mean of $0.38 \mathrm{pg}$, indicating that there is a genome, and likely a chromosome, number upper limit.

Keywords: chromosome evolution; cell biology; genome size; karyotype; ants; genome evolution 


\section{Introduction}

The genome of any organism is organized into chromosomes that can vary in size, morphology, and DNA composition. Changes in the structure of the chromosomes via mutations and rearrangements, or via numerical changes (aneuploidy) due to errors in the spindle during cell division, can contribute to speciation by impairing gene flow and decreasing fitness [1]. Although there is ample evidence that chromosome changes can contribute to lineage diversification (see [2]), questions and debates remain about the circumstances. However, as morphological traits, chromosomal features are a reliable criterion in evolutionary and taxonomic studies. Among insects, ants display a tremendous diversity of chromosome numbers, varying from $n=1$ to $n=60$ (reviewed in [2,3]). This large range in chromosome number attracted the attention of geneticists and raised numerous questions concerning the mechanisms of chromosome evolution in Formicidae. Different authors have described several evolutionary pathways that could better explain the chromosome changes in ants (see [4,5]). Despite the diversity, no more than five percent of the taxonomically described ant species has been studied cytogenetically. The subfamily Myrmicinae contains the highest number of ant karyotypes known to date (see [3]), where the tribe Attini, sub-tribe Attina (fungus-farming ants), covers the widest spectra of the phylogeny. The known fungus-farming ant chromosome numbers range from $n=4$ to $n=27$ [3]. Due to advances in microscopy and molecular methodology in the last century, the cytogenetic studies in Formicidae have flourished resulting in more detailed descriptions using sophisticated banding methods and combining molecular phylogenies [6]. Previous studies that have evaluated fungus-farming ants cytogenetically were limited to chromosome number determination, and sometimes, due to the peculiarities of sampling, the analysis of a few individual species, not allowing for the evaluation of the relationships among species within a genus or higher-level taxa [7,8].

Detailed karyotype descriptions (which include the chromosome morphology, size, number, and banding patterns), chromosome counts, and their comparative analyses are important independent tools for use in taxonomy and in understanding chromosome evolution, particularly when relying on the phylogenetic tree [9]. The advances in molecular phylogenetic analysis and the availability of DNA data in public platforms, such as GenBank, have greatly opened up possibilities in the study of chromosomal evolution by inferring evolutionary pathways through combining phylogeny and cytogenetic data in an integrative framework $[10,11]$. Sequences of protein-coding nuclear genes are useful for resolving phylogenetic relationships within genera and between related species in fungusgrowing ants [12], and have been corroborated by inferences based on genomic data [13]. Analysis based on multi-locus data led to a recent molecular phylogenetic hypothesis that resulted in taxonomic changes at the genus level [14,15].

Beyond the number of DNA molecules, estimating the genome content of cell nuclei has contributed to our understanding of genomes through the tree of life. Recently, the nuclear content of approximately 100 ant species was estimated using flow cytometry [16], and now more genome size (GS) ant data has become available. This independent and primary genomic information has provided noteworthy data that can be coupled with cytogenetic data to shed light on the general patterns and processes of the chromosomal changes in fungus-farming ants. Further, cytogenetic and flow cytometry integrated into time-calibrated phylogeny can provide accurate information about the mode and tempo of karyotype evolution. Cytogenetic information, coupled with molecular data, allowed for recent revisionary studies, the identification of sibling species [17], and even the description of a new genus [15].

In this study, we first describe five new karyotypes of fungus-farming ants: Apterostigma madidiense (Weber, 1938), Mycocepurus goeldii (Forel, 1893), Sericomyrmex parvulus (Forel, 1912), Cyphomyrmex transversus (Emery, 1894), and Myrmicocrypta sp., based on karyomorphometric analysis. We added these karyotypes to the available data on fungusfarming ants in the Ant Chromosome Database (www.ants.ufop.br, accessed on 30 November 2021), depicting the heterochromatic banding pattern and chromosome morphology. 
We further compiled the genome size information available for the species. All these data were scattered through a time-calibrated phylogeny to evaluate the mode and tempo of chromosomal evolution in fungus-farming ants. We evaluate and discuss our results with regard to chromosome number, morphology, and genome size variation under a phylogenetic framework with a focus on the question: Is there an upper limit of chromosome number for fungus-farming ants? Our intention was not to determine a specific upper number, but to discuss and propose an indication for a limit, considering the GS estimates of ants and the outcomes of the minimum interaction theory. We further discuss the importance of cytogenetic studies.

\section{Materials and Methods}

\subsection{Colony Sampling}

Colonies were sampled from distinct Brazilian states: A. madidiense from Cachoeira do Campo, Minas Gerais (MG); S. parvulus from Marliéria, Minas Gerais (MG) (S 19² $43^{\prime} 21^{\prime \prime}$, W $42^{\circ} 43^{\prime} 26^{\prime \prime}$ ); C. transversus and Myrmicocripta sp. from Cabo Frio, Rio de Janeiro (RJ) (S $22^{\circ} 54^{\prime} 29.8^{\prime \prime}, \mathrm{W} 42^{\circ} 02^{\prime} 14.7^{\prime \prime}$ ); and M. goeldii from Morro dos Conventos, Araranguá-Santa Catarina (SC) (S $\left.28^{\circ} 56^{\prime} 08.2^{\prime \prime}, \mathrm{W} 49^{\circ} 21^{\prime} 28^{\prime \prime}\right)$. The sample collection was authorized by the ICMBio (the Chico Mendes Institute for Biodiversity Conservation: special permit 60019). All colonies were excavated and transferred to the Laboratório de Genética Evolutiva e de Populações of the Universidade Federal de Ouro Preto-MG and kept according to the protocols described by Cardoso et al. [18]. The maintenance of the colonies was required to obtain the broods used in the cytogenetic analysis.

\subsection{Chromosome Preparation and Karyomorphometry}

The chromosome slides were obtained from 20 individuals following the protocol described by Imai et al. [4], with small modifications concerning the incubation time, as described by Cardoso et al. [19]. Only high-grade metaphase slides were submitted to conventional staining with $4 \%$ Giemsa diluted in Sørensen's buffer solution at $\mathrm{pH} 6.8$. At least 10 metaphases were photographed using an Olympus BX51 microscope equipped with a digital camera (Olympus DP73). These metaphase chromosomes were evaluated to determine the chromosome number and morphology, as well the karyomorphometric data. Based on the centromere position, the morphology of the chromosomes was sorted following the nomenclature established by Levan et al. [20] as follows: telocentric, subtelocentric, submetacentric, and metacentric, the acronyms for which are $\mathrm{t}, \mathrm{st}$, sm, and $\mathrm{m}$, respectively. The karyomorphometric data was obtained by taking measurements from 10 spread metaphases with a clear centromere, chromosomal integrity, and without any overlapping, following the standardized protocol described by Cristiano et al. [21]. The chromosomal features evaluated included the entire length of each chromosome (TL), the length of the long arm of each chromosome (L), the length of the short arm of each chromosome (S), the ratio between the long and short arms $(r=\mathrm{L} / \mathrm{S})$, and the proportional contribution of each chromosome length (RL) in relation to the total length of all chromosomes (TL $\left.\times 100 / \sum T L\right)$. The distribution pattern of heterochromatin was obtained using the BSG (barium hydroxide/saline/Giemsa) banding technique following the method described by Sumner [22], with modifications for the duration of treatment with $\mathrm{Ba}(\mathrm{OH})_{2}$.

\subsection{Cytogenetic Data Compilation and Phylogenetic Analysis}

The Ant Chromosome Database (ACdb) (www.ants.ufop.br, accessed on 30 November 2021 [3]) compiles all the information regarding ant chromosome counts and karyotypes published over time, and is regularly updated. We retrieved all fungus-farming ant chromosome counts assembled in the database using the following cytogenetic parameters: diploid chromosome number $(2 n)$, haploid chromosome number $(n)$, and karyotype or karyotypic formulae. The karyotype provides the number of each type of chromosome, whether a chromosome is $\mathrm{m}$, sm, st, $\mathrm{t}$, or acrocentric (a). All the data, including the new counts and karyotypes described here, are presented in Table 1, together with the primary 
source (author reference). We further extracted the heterochromatic banding pattern of the karyotypes, when available, coding the presence of positive heterochromatin blocks $(+)$ or negative blocks (-) at centromeric (C), pericentromeric (PC), or interstitial (IN) positions, or along the long arm (LA) and/or short arm (SA). We further gathered the genome size information available for fungus-farming ants in Moura et al. [16].

To put the cytogenetic data in a phylogenetic context focusing on fungus-farming genera, coupling the phylogenetic relationship of the species with the karyotype information, we retrieved sequences of five nuclear genes (elongation factor 1-alpha-F1, elongation factor 1-alpha-F2, wingless, long-wavelength rhodopsin, and topoisomerase 1) from GenBank, which comprised the dataset assembled by Cristiano et al. [15]. We then inferred the calibrated phylogeny using BEAST v2.5 [23] under the fossilized birth-death (FBD) [24] model, setting an uncorrelated, log-normal relaxed clock model to describe the branch-specific substitution rates. The calibration of the parameters and fossils is described by Micolino et al. [17]. Substitution nucleotide models of molecular evolution were estimated for each partition in PartitionFinder 2 [25] (Table S1) and used in the analyses. Independent MCMC analyses were run for 100 million generations, sampling every 1000 generations. Runs were evaluated using Tracer v1.7 [26] with effective sample size (ESS) values for all parameters over 200 . The first $20 \%$ of the sampled tree topologies were discarded as burn-in, and the remaining trees were summarized using Treeannotator v2.5, after the removal of all fossils using the FullToExtantTreeConverter tool implemented in Beauti v2.5 [23]. The Figtree software [27] was utilized to visualize the final tree with credible intervals and branch labels (Figure S1).

\section{Results}

The metaphases of M. goeldii consisted of eight chromosomes in a diploid set $(2 n=8$, Figure 1), $2 \mathrm{~K}=6 \mathrm{~m}+2 \mathrm{sm}$ and the fundamental number $(\mathrm{FN})=16$ (Table 1). The metaphases of Myrmicocripta sp. consisted of 28 chromosomes in the diploid set $(2 n=28$, Figure 2), $2 \mathrm{~K}=24 \mathrm{~m}+4 \mathrm{sm}$ and FN $=56$ (Table 2). The metaphases of $C$. transversus consisted of 42 chromosomes in the diploid set $(2 n=42$, Figure 3$), 2 \mathrm{~K}=28 \mathrm{~m}+14 \mathrm{sm}$ and FN $=84$ (Table 3). Some individuals from the same colony bore a polymorphism in submetacentric pair 15 (Figure 4). The metaphases of $A$. madidiense consisted of 24 chromosomes in the diploid set $(2 n=24$, Figure 4$), 2 \mathrm{~K}=24 \mathrm{~m}$ and FN $=48$ (Table 4$)$. The metaphases of S. parvulus consisted of $2 n=50$ (Figure 5), $2 \mathrm{~K}=30 \mathrm{~m}+14 \mathrm{sm}+6$ st and $\mathrm{FN}=100$. We were not able to establish detailed karyomorphometric data for $S$. parvulus due to the limited number of high-quality spreads (below 10). The heterochromatic pattern of the species studied here are summarized in Table 5.

Taking into account the new information established here and the available cytogenetic data, there are 58 chromosome counts of fungus-farming ants (Table 5). From these, 10 are chromosome counts recorded for specimens not identified at the species level, whereas seven counts are related to the same taxa with distinct karyotypes from different populations. Nevertheless, genome size estimates are available for 31 species. The estimated GSs are all around the mean genome size of $0.38 \mathrm{pg}$, with two exceptions: Apterostigma spp. $(0.61-0.81 \mathrm{pg})$ and C. transversus (0.50 pg). Spreading the cytogenetic and GS data across the calibrated phylogeny that was generated, we detected wide chromosomal and genome size variability among the species of fungus-farming ants (Figure 6). Although limited to the number of observations, there is a clear pattern where karyotypes with a haploid number of 10 to 12 are distributed along the phylogeny, and most species bear a GS of below $0.40 \mathrm{pg}$. Higher chromosome numbers $(n>15)$ are restricted to some lineages within the genera. Nevertheless, even higher chromosome numbers $(n>25)$ are rare and restricted to lineages of Neoattini (Figure 6). Our data allow for the identification of a general pattern indicating lineages that are recently divergent, splitting below $26 \mathrm{Mya}$, and that are more homogenous with regard to the number of chromosomes (Figure 6). For instance, the known Trachymyrmex species show a haploid set of 10 chromosomes, and all Amoimyrmex and Atta show a haploid set of 11 chromosomes (Figure 6). The compiled 
heterochromatic pattern data suggest that karyotypes under 15 haploid chromosomes show heterochromatin restricted to the centromere, whereas karyotypes with a higher number of chromosomes $(n>15)$ show a higher number of positive heterochromatic blocks, including chromosomes with a heterochromatic long arm (Table 5).

(a)

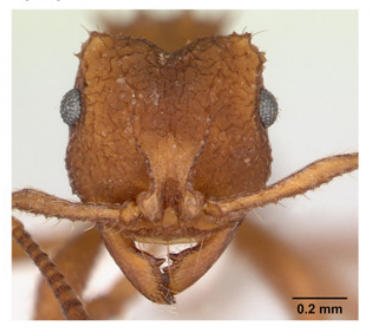

(b)

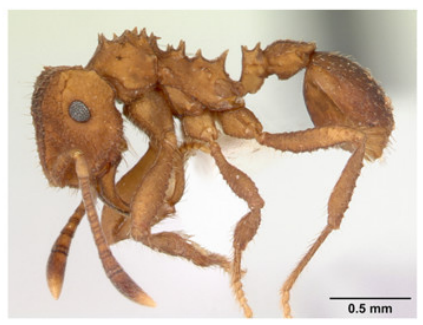

$\mathrm{m}$
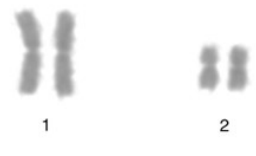

$\mathrm{sm}$
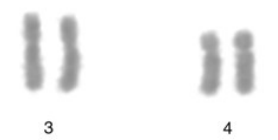

Figure 1. Mitotic chromosomes of Mycocepurus goeldii stained with Giemsa. Species (a) images and (b) diploid karyotype of Mycocepurus goeldii with $2 n=8$ chromosomes. $\mathrm{m}=$ metacentric, $\mathrm{sm}=$ submetacentric. Scale bar $=5 \mu \mathrm{m}$. Ant image from AntWeb (www.antweb.org, accessed on 13 June 2021): Mycocepurus goeldii (CASENT0173988, photo by: A. Nobile).

Table 1. Karyomorphometric analyses of the chromosomes of Mycocepurus goeldii. All measurements are given in " $\mu \mathrm{m}$ ". TL: total length; L: long arm length; S: short arm length; RL: relative length; r: arm ratio; KL: karyotype length.

\begin{tabular}{ccccccc}
\hline $\begin{array}{c}\text { Chromosome } \\
\text { (Homologue) }\end{array}$ & TL $( \pm$ SD) & L $( \pm$ SD) & S \pm SD) & RL $( \pm$ SD) & r $( \pm$ SD) & Classification \\
\hline 1 & $8.79 \pm 3.04$ & $4.78 \pm 1.71$ & $4.01 \pm 1.37$ & $15.70 \pm 0.35$ & $1.19 \pm 0.13$ & Metacentric \\
\hline$(1)$ & $8.37 \pm 3.03$ & $4.48 \pm 1.6$ & $3.89 \pm 1.46$ & $14.89 \pm 0.64$ & $1.16 \pm 0.13$ & Metacentric \\
\hline 2 & $4.82 \pm 1.57$ & $2.82 \pm 1.00$ & $1.99 \pm 0.57$ & $08.66 \pm 0.37$ & $1.40 \pm 0.13$ & Metacentric \\
\hline$(2)$ & $4.64 \pm 1.52$ & $2.64 \pm 0.89$ & $2.00 \pm 0.64$ & $08.34 \pm 0.35$ & $1.32 \pm 0.12$ & Metacentric \\
\hline 3 & $8.02 \pm 3.03$ & $4.97 \pm 1.73$ & $3.05 \pm 1.38$ & $14.19 \pm 0.87$ & $1.70 \pm 0.28$ & Submetacentric \\
\hline$(3)$ & $7.76 \pm 2.98$ & $4.87 \pm 1.97$ & $2.99 \pm 1.03$ & $13.70 \pm 0.91$ & $1.61 \pm 0.23$ & Submetacentric \\
\hline 4 & $6.90 \pm 2.16$ & $4.90 \pm 1.62$ & $2.00 \pm 0.58$ & $12.47 \pm 1.04$ & $2.45 \pm 0.36$ & Submetacentric \\
\hline$(4)$ & $6.67 \pm 2.14$ & $4.65 \pm 1.50$ & $2.03 \pm 0.66$ & $12.05 \pm 0.90$ & $2.29 \pm 0.20$ & Submetacentric \\
\hline KL & 55.97 & & & & &
\end{tabular}

(a)

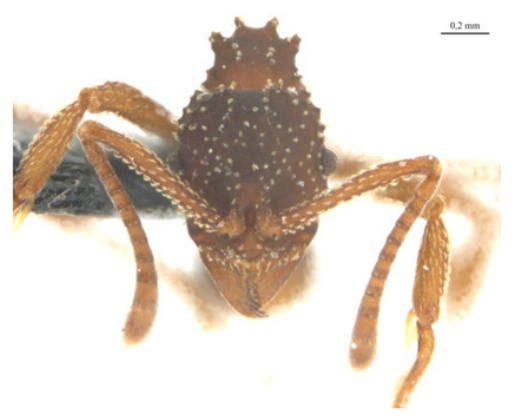

(b)

$\mathrm{m}$

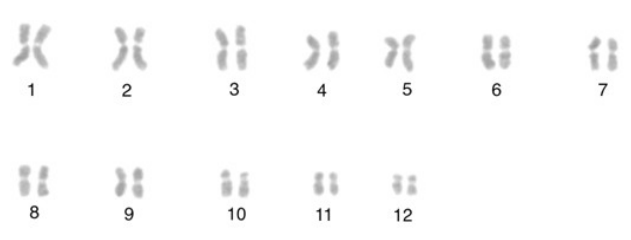

$\mathrm{sm}$

Figure 2. Mitotic chromosomes of Myrmicocripta sp. stained with Giemsa. Species (a) images and (b) diploid karyotype of Myrmicocripta sp. with $2 n=28$ chromosomes. $\mathrm{m}=$ metacentric; $\mathrm{sm}=$ submetacentric. Scale bar $=5 \mu \mathrm{m}$. Ant image was obtained in our lab. 
Table 2. Karyomorphometric analyses of the chromosomes of Myrmicocripta sp. All measurements are given in " $\mu \mathrm{m}$ ". TL: total length; L: long arm length; S: short arm length; RL: relative length; r: arm ratio; KL: karyotype length.

\begin{tabular}{|c|c|c|c|c|c|c|}
\hline $\begin{array}{l}\text { Chromosome } \\
\text { (Homologue) }\end{array}$ & TL $( \pm$ SD) & $\mathbf{L}( \pm \mathbf{S D})$ & $\mathbf{S}( \pm \mathrm{SD})$ & $\mathbf{R L}( \pm \mathrm{SD})$ & $\mathbf{r}( \pm \mathrm{SD})$ & Classification \\
\hline 1 & $4.27 \pm 0.88$ & $2.37 \pm 0.44$ & $1.89 \pm 0.46$ & $5.20 \pm 0.18$ & $1.28 \pm 0.15$ & Metacentric \\
\hline (1) & $4.14 \pm 0.89$ & $2.27 \pm 0.44$ & $1.87 \pm 0.46$ & $5.03 \pm 0.15$ & $1.23 \pm 0.13$ & Metacentric \\
\hline 2 & $3.96 \pm 0.83$ & $2.24 \pm 0.49$ & $1.72 \pm 0.38$ & $4.82 \pm 0.18$ & $1.32 \pm 0.16$ & Metacentric \\
\hline (2) & $3.89 \pm 0.81$ & $2.12 \pm 0.46$ & $1.77 \pm 0.37$ & $4.73 \pm 0.17$ & $1.21 \pm 0.10$ & Metacentric \\
\hline 3 & $3.76 \pm 0.89$ & $2.07 \pm 0.57$ & $1.69 \pm 0.38$ & $4.54 \pm 0.20$ & $1.23 \pm 0.22$ & Metacentric \\
\hline (3) & $3.68 \pm 0.89$ & $1.99 \pm 0.49$ & $1.69 \pm 0.43$ & $4.44 \pm 0.18$ & $1.19 \pm 0.14$ & Metacentric \\
\hline 4 & $3.44 \pm 0.79$ & $1.95 \pm 0.50$ & $1.49 \pm 0.31$ & $4.16 \pm 0.14$ & $1.30 \pm 0.13$ & Metacentric \\
\hline (4) & $3.30 \pm 0.75$ & $1.83 \pm 0.39$ & $1.47 \pm 0.37$ & $4.00 \pm 0.12$ & $1.26 \pm 0.10$ & Metacentric \\
\hline 5 & $3.22 \pm 0.71$ & $1.79 \pm 0.38$ & $1.43 \pm 0.34$ & $3.91 \pm 0.11$ & $1.26 \pm 0.11$ & Metacentric \\
\hline (5) & $3.14 \pm 0.71$ & $1.70 \pm 0.34$ & $1.43 \pm 0.37$ & $3.80 \pm 0.09$ & $1.21 \pm 0.11$ & Metacentric \\
\hline 6 & $3.06 \pm 0.69$ & $1.69 \pm 0.37$ & $1.37 \pm 0.34$ & $3.70 \pm 0.10$ & $1.25 \pm 0.15$ & Metacentric \\
\hline (6) & $2.99 \pm 0.65$ & $1.68 \pm 0.37$ & $1.31 \pm 0.31$ & $3.63 \pm 0.06$ & $1.29 \pm 0.13$ & Metacentric \\
\hline 7 & $2.90 \pm 0.63$ & $1.62 \pm 0.35$ & $1.28 \pm 0.31$ & $3.51 \pm 0.08$ & $1.28 \pm 0.14$ & Metacentric \\
\hline (7) & $2.86 \pm 0.64$ & $1.57 \pm 0.31$ & $1.29 \pm 0.33$ & $3.46 \pm 0.07$ & $1.24 \pm 0.12$ & Metacentric \\
\hline 8 & $2.80 \pm 0.64$ & $1.57 \pm 0.35$ & $1.24 \pm 0.31$ & $3.39 \pm 0.06$ & $1.29 \pm 0.14$ & Metacentric \\
\hline (8) & $2.73 \pm 0.62$ & $1.54 \pm 0.34$ & $1.20 \pm 0.29$ & $3.31 \pm 0.10$ & $1.30 \pm 0.14$ & Metacentric \\
\hline 9 & $2.65 \pm 0.63$ & $1.47 \pm 0.31$ & $1.17 \pm 0.32$ & $3.20 \pm 0.14$ & $1.28 \pm 0.16$ & Metacentric \\
\hline (9) & $2.56 \pm 0.63$ & $1.41 \pm 0.33$ & $1.15 \pm 0.31$ & $3.09 \pm 0.16$ & $1.25 \pm 0.17$ & Metacentric \\
\hline 10 & $2.41 \pm 0.57$ & $1.39 \pm 0.29$ & $1.01 \pm 0.30$ & $2.91 \pm 0.17$ & $1.42 \pm 0.20$ & Metacentric \\
\hline$(10)$ & $2.20 \pm 0.55$ & $1.22 \pm 0.31$ & $0.97 \pm 0.30$ & $2.65 \pm 0.20$ & $1.37 \pm 0.16$ & Metacentric \\
\hline 11 & $1.94 \pm 0.48$ & $1.11 \pm 0.27$ & $0.82 \pm 0.25$ & $2.33 \pm 0.11$ & $1.43 \pm 0.17$ & Metacentric \\
\hline (11) & $1.84 \pm 0.44$ & $1.06 \pm 0.26$ & $0.79 \pm 0.20$ & $2.22 \pm 0.10$ & $1.36 \pm 0.17$ & Metacentric \\
\hline 12 & $1.70 \pm 0.39$ & $0.98 \pm 0.22$ & $0.72 \pm 0.18$ & $2.06 \pm 0.11$ & $1.38 \pm 0.13$ & Metacentric \\
\hline (12) & $1.62 \pm 0.38$ & $0.93 \pm 0.21$ & $0.69 \pm 0.17$ & $1.95 \pm 0.09$ & $1.35 \pm 0.13$ & Metacentric \\
\hline 13 & $3.57 \pm 0.82$ & $2.39 \pm 0.53$ & $1.17 \pm 0.31$ & $4.34 \pm 0.46$ & $2.07 \pm 0.26$ & Submetacentric \\
\hline (13) & $3.26 \pm 0.85$ & $2.19 \pm 0.53$ & $1.07 \pm 0.31$ & $3.99 \pm 0.63$ & $2.08 \pm 0.11$ & Submetacentric \\
\hline 14 & $2.36 \pm 0.50$ & $1.67 \pm 0.39$ & $0.70 \pm 0.12$ & $2.88 \pm 0.20$ & $2.38 \pm 0.30$ & Submetacentric \\
\hline (14) & $2.22 \pm 0.43$ & $1.52 \pm 0.31$ & $0.70 \pm 0.15$ & $2.72 \pm 0.22$ & $2.18 \pm 0.30$ & Submetacentric \\
\hline KL & 82.47 & & & & & \\
\hline
\end{tabular}


(a)

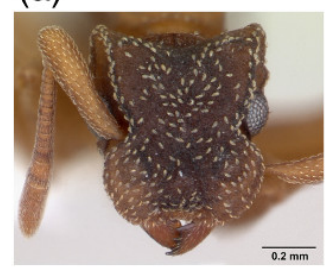

(b)

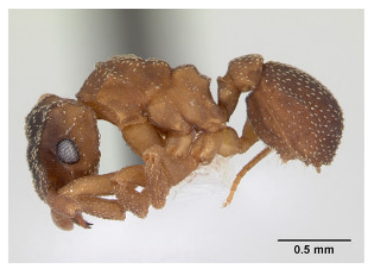

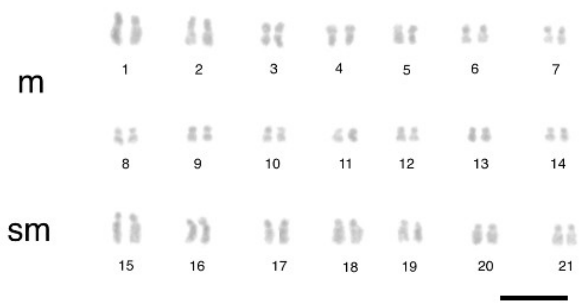

(c)

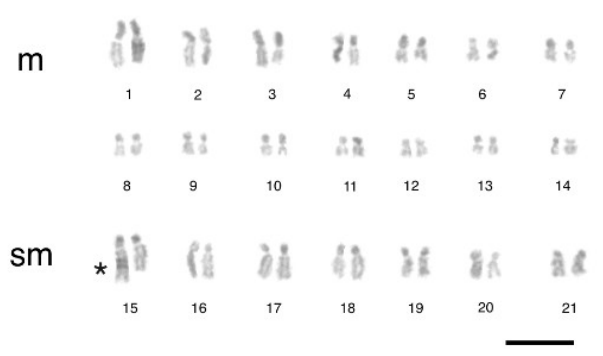

Figure 3. Mitotic chromosomes of Cyphomyrmex transversus stained with Giemsa. Species (a) images and (b,c) diploid karyotype of Cyphomyrmex transversus with $2 n=42$ chromosomes. $\mathrm{m}=$ metacentric, $\mathrm{sm}=$ submetacentric. Polymorphism in chromosome pair 15 is marked by an asterisk. Scale bar $=5 \mu \mathrm{m}$. Ant image from AntWeb (www.antweb.org, accessed on 13 June 2021): Cyphomyrmex transversus (CASENT0173958, photo by: A. Nobile).

Table 3. Karyomorphometric analyses of the chromosomes of Cyphomyrmex transversus. All measurements are given in " $\mu \mathrm{m}$ ". TL: total length; L: long arm length; S: short arm length; RL: relative length; r: arm ratio; KL: karyotype length.

\begin{tabular}{|c|c|c|c|c|c|c|}
\hline $\begin{array}{l}\text { Chromosome } \\
\text { (Homologue) }\end{array}$ & TL ( \pm SD) & $\mathbf{L}( \pm \mathbf{S D})$ & $\mathbf{S}( \pm \mathrm{SD})$ & $\mathbf{R L}( \pm \mathbf{S D})$ & $\mathbf{r}( \pm \mathrm{SD})$ & Classification \\
\hline 1 & $3.19 \pm 1.40$ & $1.76 \pm 0.73$ & $1.43 \pm 0.68$ & $3.84 \pm 0.2$ & $1.27 \pm 0.14$ & Metacentric \\
\hline (1) & $2.91 \pm 1.29$ & $1.71 \pm 0.76$ & $1.20 \pm 0.55$ & $3.50 \pm 0.22$ & $1.45 \pm 0.22$ & Metacentric \\
\hline 2 & $2.62 \pm 1.06$ & $1.40 \pm 0.62$ & $1.22 \pm 0.47$ & $3.18 \pm 0.18$ & $1.18 \pm 0.12$ & Metacentric \\
\hline$(2)$ & $2.48 \pm 1.09$ & $1.31 \pm 0.62$ & $1.17 \pm 0.50$ & $2.98 \pm 0.23$ & $1.28 \pm 0.16$ & Metacentric \\
\hline 3 & $2.39 \pm 1.04$ & $1.28 \pm 0.53$ & $1.10 \pm 0.52$ & $2.87 \pm 0.24$ & $1.22 \pm 0.17$ & Metacentric \\
\hline (3) & $2.23 \pm 0.94$ & $1.31 \pm 0.53$ & $0.92 \pm 0.43$ & $2.69 \pm 0.21$ & $1.44 \pm 0.22$ & Metacentric \\
\hline 4 & $2.13 \pm 0.92$ & $1.26 \pm 0.59$ & $0.86 \pm 0.33$ & $2.56 \pm 0.14$ & $1.44 \pm 0.20$ & Metacentric \\
\hline (4) & $2.08 \pm 0.89$ & $1.18 \pm 0.5$ & $0.90 \pm 0.40$ & $2.51 \pm 0.10$ & $1.36 \pm 0.19$ & Metacentric \\
\hline 5 & $2.00 \pm 0.84$ & $1.13 \pm 0.43$ & $0.88 \pm 0.43$ & $2.42 \pm 0.09$ & $1.34 \pm 0.26$ & Metacentric \\
\hline (5) & $1.89 \pm 0.71$ & $1.10 \pm 0.42$ & $0.79 \pm 0.30$ & $2.32 \pm 0.14$ & $1.40 \pm 0.13$ & Metacentric \\
\hline 6 & $1.83 \pm 0.67$ & $1.03 \pm 0.37$ & $0.80 \pm 0.33$ & $2.24 \pm 0.12$ & $1.32 \pm 0.23$ & Metacentric \\
\hline (6) & $1.80 \pm 0.65$ & $0.97 \pm 0.31$ & $0.82 \pm 0.36$ & $2.20 \pm 0.11$ & $1.26 \pm 0.13$ & Metacentric \\
\hline 7 & $1.75 \pm 0.65$ & $0.97 \pm 0.37$ & $0.79 \pm 0.28$ & $2.15 \pm 0.10$ & $1.25 \pm 0.16$ & Metacentric \\
\hline (7) & $1.72 \pm 0.63$ & $0.95 \pm 0.35$ & $0.77 \pm 0.30$ & $2.11 \pm 0.08$ & $1.34 \pm 0.12$ & Metacentric \\
\hline 8 & $1.68 \pm 0.63$ & $0.98 \pm 0.33$ & $0.72 \pm 0.29$ & $2.05 \pm 0.11$ & $1.39 \pm 0.14$ & Metacentric \\
\hline (8) & $1.66 \pm 0.62$ & $0.94 \pm 0.37$ & $0.72 \pm 0.27$ & $2.03 \pm 0.11$ & $1.36 \pm 0.18$ & Metacentric \\
\hline 9 & $1.62 \pm 0.60$ & $0.95 \pm 0.38$ & $0.68 \pm 0.26$ & $1.99 \pm 0.08$ & $1.42 \pm 0.22$ & Metacentric \\
\hline (9) & $1.59 \pm 0.60$ & $0.91 \pm 0.37$ & $0.68 \pm 0.26$ & $1.95 \pm 0.10$ & $1.42 \pm 0.18$ & Metacentric \\
\hline 10 & $1.55 \pm 0.60$ & $0.89 \pm 0.36$ & $0.66 \pm 0.25$ & $1.89 \pm 0.08$ & $1.33 \pm 0.13$ & Metacentric \\
\hline (10) & $1.54 \pm 0.60$ & $0.85 \pm 0.30$ & $0.69 \pm 0.29$ & $1.88 \pm 0.07$ & $1.27 \pm 0.13$ & Metacentric \\
\hline
\end{tabular}


Table 3. Cont.

\begin{tabular}{|c|c|c|c|c|c|c|}
\hline $\begin{array}{l}\text { Chromosome } \\
\text { (Homologue) }\end{array}$ & TL $( \pm S D)$ & $\mathbf{L}( \pm \mathrm{SD})$ & $\mathbf{S}( \pm \mathbf{S D})$ & $\mathbf{R L}( \pm \mathbf{S D})$ & $\mathbf{r}( \pm \mathrm{SD})$ & Classification \\
\hline 11 & $1.52 \pm 0.56$ & $0.82 \pm 0.28$ & $0.70 \pm 0.28$ & $1.86 \pm 0.08$ & $1.21 \pm 0.15$ & Metacentric \\
\hline (11) & $1.48 \pm 0.55$ & $0.83 \pm 0.30$ & $0.66 \pm 0.25$ & $1.82 \pm 0.07$ & $1.26 \pm 0.08$ & Metacentric \\
\hline 12 & $1.45 \pm 0.50$ & $0.85 \pm 0.31$ & $0.60 \pm 0.20$ & $1.79 \pm 0.10$ & $1.43 \pm 0.16$ & Metacentric \\
\hline (12) & $1.41 \pm 0.50$ & $0.81 \pm 0.28$ & $0.60 \pm 0.22$ & $1.74 \pm 0.10$ & $1.38 \pm 0.14$ & Metacentric \\
\hline 13 & $1.38 \pm 0.49$ & $0.78 \pm 0.26$ & $0.59 \pm 0.24$ & $1.69 \pm 0.08$ & $1.37 \pm 0.21$ & Metacentric \\
\hline (13) & $1.37 \pm 0.49$ & $0.77 \pm 0.25$ & $0.59 \pm 0.24$ & $1.68 \pm 0.08$ & $1.34 \pm 0.15$ & Metacentric \\
\hline 14 & $1.34 \pm 0.49$ & $0.75 \pm 0.28$ & $0.59 \pm 0.21$ & $1.64 \pm 0.09$ & $1.28 \pm 0.10$ & Metacentric \\
\hline (14) & $1.27 \pm 0.44$ & $0.76 \pm 0.29$ & $0.51 \pm 0.16$ & $1.56 \pm 0.11$ & $1.46 \pm 0.14$ & Metacentric \\
\hline 15 & $2.72 \pm 1.32$ & $1.90 \pm 0.95$ & $0.82 \pm 0.38$ & $3.29 \pm 0.73$ & $2.28 \pm 0.19$ & Submetacentric \\
\hline (15) & $2.58 \pm 1.20$ & $1.80 \pm 0.84$ & $0.78 \pm 0.37$ & $3.14 \pm 0.71$ & $2.33 \pm 0.28$ & Submetacentric \\
\hline 16 & $2.60 \pm 1.00$ & $1.80 \pm 0.67$ & $0.80 \pm 0.33$ & $3.18 \pm 0.15$ & $2.27 \pm 0.17$ & Submetacentric \\
\hline (16) & $2.49 \pm 0.93$ & $1.75 \pm 0.69$ & $0.74 \pm 0.25$ & $3.06 \pm 0.14$ & $2.32 \pm 0.21$ & Submetacentric \\
\hline 17 & $2.43 \pm 0.94$ & $1.69 \pm 0.64$ & $0.75 \pm 0.31$ & $2.97 \pm 0.07$ & $2.27 \pm 0.18$ & Submetacentric \\
\hline (17) & $2.34 \pm 0.98$ & $1.62 \pm 0.65$ & $0.72 \pm 0.33$ & $2.84 \pm 0.09$ & $2.29 \pm 0.15$ & Submetacentric \\
\hline 18 & $2.18 \pm 0.95$ & $1.48 \pm 0.62$ & $0.71 \pm 0.33$ & $2.64 \pm 0.16$ & $2.11 \pm 0.16$ & Submetacentric \\
\hline (18) & $2.13 \pm 0.94$ & $1.47 \pm 0.68$ & $0.66 \pm 0.26$ & $2.56 \pm 0.15$ & $2.21 \pm 0.25$ & Submetacentric \\
\hline 19 & $2.04 \pm 0.93$ & $1.39 \pm 0.59$ & $0.65 \pm 0.35$ & $2.45 \pm 0.17$ & $2.19 \pm 0.21$ & Submetacentric \\
\hline (19) & $2.00 \pm 0.90$ & $1.35 \pm 0.61$ & $0.66 \pm 0.30$ & $2.41 \pm 0.18$ & $2.07 \pm 0.21$ & Submetacentric \\
\hline 20 & $1.91 \pm 0.78$ & $1.27 \pm 0.50$ & $0.64 \pm 0.29$ & $2.32 \pm 0.11$ & $2.01 \pm 0.16$ & Submetacentric \\
\hline (20) & $1.82 \pm 0.76$ & $1.24 \pm 0.54$ & $0.59 \pm 0.23$ & $2.21 \pm 0.17$ & $2.08 \pm 0.26$ & Submetacentric \\
\hline 21 & $1.64 \pm 0.75$ & $1.10 \pm 0.52$ & $0.54 \pm 0.23$ & $1.99 \pm 0.25$ & $2.02 \pm 0.17$ & Submetacentric \\
\hline (21) & $1.52 \pm 0.75$ & $1.03 \pm 0.55$ & $0.50 \pm 0.20$ & $1.83 \pm 0.22$ & $2.00 \pm 0.21$ & Submetacentric \\
\hline KL & 82.28 & & & & & \\
\hline
\end{tabular}

(a)

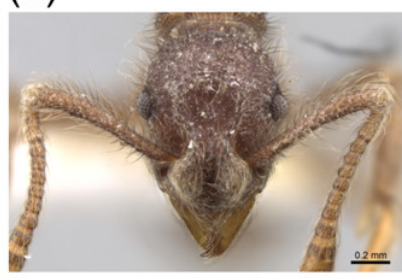

(b)

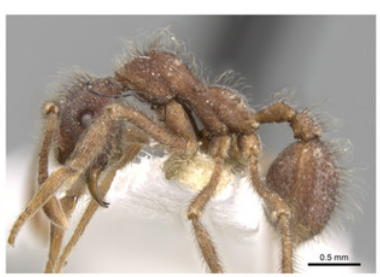

m

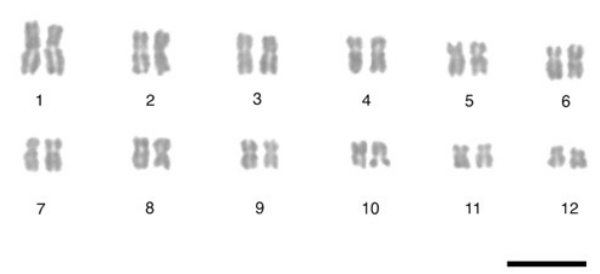

Figure 4. Mitotic chromosomes of Apterostigma madidiense stained with Giemsa. Species (a) images and (b) diploid karyotype of Apterostigma madidiense with $2 n=24$ chromosomes. $\mathrm{m}=$ metacentric. Scale bar $=5 \mu \mathrm{m}$. Ant image from AntWeb (www.antweb.org, accessed on 13 June 2021): Apterostigma madidiense (CASENT0281778, photo by: S. Hartman). 
Table 4. Karyomorphometric analyses of the chromosomes of Apterostigma madidiense. All measurements are given in " $\mu$ m". TL: total length; L: long arm length; S: short arm length; RL: relative length; r: arm ratio; KL: karyotype length.

\begin{tabular}{|c|c|c|c|c|c|c|}
\hline $\begin{array}{l}\text { Chromosome } \\
\text { (Homologue) }\end{array}$ & TL $( \pm$ SD) & $\mathbf{L}( \pm \mathrm{SD})$ & $\mathbf{S}( \pm \mathrm{SD})$ & $\mathbf{R L}( \pm \mathbf{S D})$ & $\mathbf{r}( \pm \mathrm{SD})$ & Classification \\
\hline 1 & $6.95 \pm 1.32$ & $3.73 \pm 0.81$ & $3.22 \pm 0.52$ & $6.74 \pm 0.25$ & $1.17 \pm 0.09$ & Metacentric \\
\hline (1) & $6.58 \pm 1.21$ & $3.54 \pm 0.69$ & $3.06 \pm 0.57$ & $6.39 \pm 0.24$ & $1.17 \pm 0.07$ & Metacentric \\
\hline 2 & $5.75 \pm 1.12$ & $3.13 \pm 0.63$ & $2.62 \pm 0.46$ & $5.58 \pm 0.23$ & $1.19 \pm 0.07$ & Metacentric \\
\hline$(2)$ & $5.39 \pm 1.05$ & $2.92 \pm 0.54$ & $2.45 \pm 0.52$ & $5.22 \pm 0.18$ & $1.20 \pm 0.13$ & Metacentric \\
\hline 3 & $5.01 \pm 0.89$ & $2.77 \pm 0.44$ & $2.24 \pm 0.48$ & $4.87 \pm 0.15$ & $1.26 \pm 0.18$ & Metacentric \\
\hline (3) & $4.82 \pm 0.86$ & $2.79 \pm 0.49$ & $2.03 \pm 0.39$ & $4.68 \pm 0.13$ & $1.38 \pm 0.18$ & Metacentric \\
\hline 4 & $4.71 \pm 0.82$ & $2.61 \pm 0.43$ & $2.09 \pm 0.53$ & $4.58 \pm 0.12$ & $1.36 \pm 0.16$ & Metacentric \\
\hline (4) & $4.60 \pm 0.79$ & $2.63 \pm 0.41$ & $1.97 \pm 0.56$ & $4.47 \pm 0.09$ & $1.48 \pm 0.15$ & Metacentric \\
\hline 5 & $4.50 \pm 0.74$ & $2.54 \pm 0.32$ & $1.94 \pm 0.44$ & $4.38 \pm 0.08$ & $1.35 \pm 0.19$ & Metacentric \\
\hline (5) & $4.38 \pm 0.72$ & $2.54 \pm 0.35$ & $1.83 \pm 0.41$ & $4.26 \pm 0.07$ & $1.42 \pm 0.21$ & Metacentric \\
\hline 6 & $4.31 \pm 0.73$ & $2.56 \pm 0.40$ & $1.73 \pm 0.36$ & $4.19 \pm 0.07$ & $1.50 \pm 0.17$ & Metacentric \\
\hline (6) & $4.25 \pm 0.71$ & $2.36 \pm 0.47$ & $1.86 \pm 0.47$ & $4.14 \pm 0.07$ & $1.43 \pm 0.25$ & Metacentric \\
\hline 7 & $4.20 \pm 0.71$ & $2.47 \pm 0.32$ & $1.75 \pm 0.42$ & $4.09 \pm 0.07$ & $1.45 \pm 0.22$ & Metacentric \\
\hline (7) & $4.12 \pm 0.72$ & $2.36 \pm 0.38$ & $1.77 \pm 0.38$ & $4.01 \pm 0.10$ & $1.35 \pm 0.20$ & Metacentric \\
\hline 8 & $4.02 \pm 0.69$ & $2.29 \pm 0.40$ & $1.72 \pm 0.35$ & $3.91 \pm 0.08$ & $1.35 \pm 0.21$ & Metacentric \\
\hline (8) & $3.84 \pm 0.62$ & $2.09 \pm 0.50$ & $1.75 \pm 0.22$ & $3.74 \pm 0.12$ & $1.31 \pm 0.19$ & Metacentric \\
\hline 9 & $3.64 \pm 0.61$ & $2.06 \pm 0.34$ & $1.57 \pm 0.29$ & $3.54 \pm 0.17$ & $1.33 \pm 0.15$ & Metacentric \\
\hline (9) & $3.48 \pm 0.54$ & $1.96 \pm 0.31$ & $1.53 \pm 0.27$ & $3.40 \pm 0.14$ & $1.30 \pm 0.19$ & Metacentric \\
\hline 10 & $3.35 \pm 0.52$ & $1.94 \pm 0.33$ & $1.42 \pm 0.24$ & $3.27 \pm 0.10$ & $1.38 \pm 0.19$ & Metacentric \\
\hline$(10)$ & $3.22 \pm 0.46$ & $1.83 \pm 0.30$ & $1.40 \pm 0.23$ & $3.15 \pm 0.13$ & $1.32 \pm 0.23$ & Metacentric \\
\hline 11 & $3.12 \pm 0.46$ & $1.81 \pm 0.21$ & $1.31 \pm 0.27$ & $3.05 \pm 0.12$ & $1.41 \pm 0.18$ & Metacentric \\
\hline (11) & $3.06 \pm 0.47$ & $1.83 \pm 0.28$ & $1.23 \pm 0.20$ & $2.98 \pm 0.11$ & $1.50 \pm 0.12$ & Metacentric \\
\hline 12 & $2.82 \pm 0.48$ & $1.63 \pm 0.31$ & $1.20 \pm 0.19$ & $2.76 \pm 0.22$ & $1.36 \pm 0.18$ & Metacentric \\
\hline (12) & $2.64 \pm 0.33$ & $1.53 \pm 0.20$ & $1.12 \pm 0.14$ & $2.59 \pm 0.19$ & $1.36 \pm 0.13$ & Metacentric \\
\hline KL & 102.76 & & & & & \\
\hline
\end{tabular}

(a)

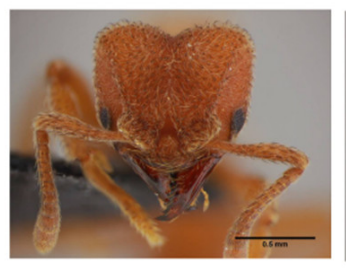

(b)

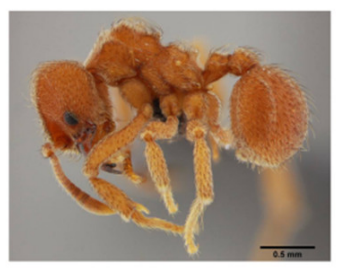

m

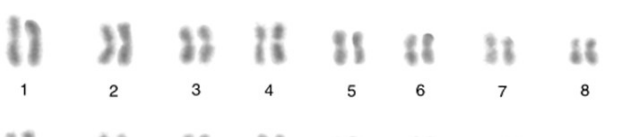

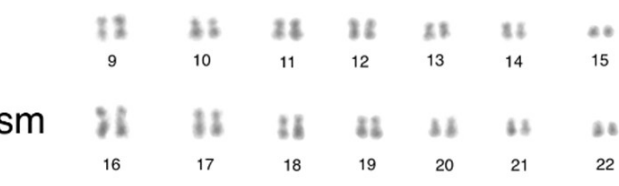

st $\begin{array}{lll}\text { if } & \text { b2 } & \text { is } \\ 23 & 24 & 25\end{array}$

Figure 5. Mitotic chromosomes of Sericomyrmex parvulus stained with Giemsa. Species (a) images and (b) diploid karyotype of Sericomyrmex parvulus with $2 n=50$ chromosomes. $\mathrm{m}=$ metacentric; $\mathrm{sm}=$ submetacentric; st $=$ subtelocentric. Scale bar $=5 \mu \mathrm{m}$. Ant image from AntWeb (www.antweb.org, accessed on 13 June 2021): Sericomyrmex parvulus (UFV-LABECOL-000372, photo by: J. Chaul). 


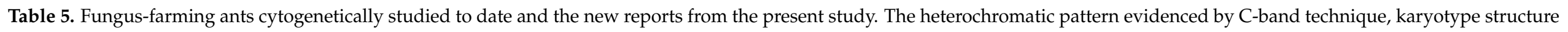
(formula), and genome size is highlighted.

\begin{tabular}{|c|c|c|c|c|c|c|c|c|c|c|c|}
\hline \multirow[t]{2}{*}{ Species Studied } & \multirow[t]{2}{*}{$2 n(n)$} & \multirow[t]{2}{*}{$\begin{array}{c}\text { Genome } \\
\text { Size 1C } \\
\text { (pg) }\end{array}$} & \multirow[t]{2}{*}{ Local/State } & \multirow[t]{2}{*}{ Country } & \multirow[t]{2}{*}{ Karyotype } & \multicolumn{5}{|c|}{$\begin{array}{c}\text { Heterochromatic } \\
\text { Pattern } \\
\text { (C-Bands) }\end{array}$} & \multirow[t]{2}{*}{ References } \\
\hline & & & & & & $\mathrm{C}$ & PC & IN & SA & LA & \\
\hline Acromyrmex ambiguus & 38 & 0.33 & $\mathrm{SP}$ & Uruguay & $\begin{array}{c}14 m+12 s m+8 s t+4 a(2 m+ \\
6 s m+16 s t+14 a)\end{array}$ & + & + & - & + & - & {$[28,29]$} \\
\hline Acromyrmex ameliae & 36 & - & MG & Brazil & $10 m+16 s m+8 s t+2 a$ & + & - & - & + & - & {$[30]$} \\
\hline Acromyrmex aspersus & 38 & - & MG & Brazil & $8 m+10 s m+16 s t+4 a$ & & & & & & {$[31]$} \\
\hline Acromyrmex balzani & 38 & 0.37 & MG & Brazil, French Guiana & $12 \mathrm{~m}+10 \mathrm{sm}+14 \mathrm{st}+2 \mathrm{a}$ & + & + & - & + & - & {$[32,33]$} \\
\hline Acromyrmex coronatus & $38(19)$ & 0.34 & MG & Brazil & $12 m+8 s m+16 s t+2 a$ & + & + & - & + & - & {$[32]$} \\
\hline Acromyrmex crassispinus & 38 & 0.34 & MG & Brazil & $12 m+20 s m+4 s t+2 a$ & & & & & & {$[29,34]$} \\
\hline Acromyrmex disciger & 38 & 0.33 & MG & Brazil & $10 \mathrm{~m}+12 \mathrm{sm}+14 \mathrm{st}+2 \mathrm{a}$ & + & + & - & + & - & [32] \\
\hline Acromyrmex echinatior & 38 & 0.36 & & Panama & $8 m+6 s m+14 s t+10 a$ & - & - & + & + & - & {$[32]$} \\
\hline Acromyrmex heyeri & 38 & - & RS & Uruguay, Brazil & $2 m+6 s m+16 s t+14 a$ & & & & & & {$[28,35]$} \\
\hline Acromyrmex hispidus & 38 & - & & Uruguay & $2 m+6 s m+16 s t+14 a$ & & & & & & [28] \\
\hline Acromyrmex niger & 38 & 0.36 & MG & Brazil & $12 \mathrm{~m}+14 \mathrm{sm}+10 \mathrm{st}+2 \mathrm{a}$ & - & + & - & + & - & {$[32]$} \\
\hline Acromyrmex nigrosetosus & $38(19)$ & 0.35 & MG & Brazil & $12 \mathrm{~m}+14 \mathrm{sm}+10 \mathrm{st}+2 \mathrm{a}$ & & & & & & [29] \\
\hline Acromyrmex rugosus & 38 & 0.35 & MG & Brazil & $16 \mathrm{~m}+12 \mathrm{sm}+8 \mathrm{st}+2 \mathrm{a}$ & + & + & - & + & - & [32] \\
\hline $\begin{array}{c}\text { Acromyrmex subterraneus } \\
\text { molestans }\end{array}$ & 38 & 0.34 & MG & Brazil & $10 m+10 s m+16 s t+2 a$ & & & & & & {$[31,34]$} \\
\hline $\begin{array}{c}\text { Acromyrmex subterraneus } \\
\text { subterraneus }\end{array}$ & 38 & 0.35 & MG & Brazil & $14 \mathrm{~m}+18 \mathrm{sm}+4 \mathrm{st}+2 \mathrm{a}$ & + & + & - & + & - & {$[29,34]$} \\
\hline $\begin{array}{c}\text { Acromyrmex subterraneus } \\
\text { brunneus }\end{array}$ & 38 & 0.34 & MG & Brazil & $10 m+14 s m+12 s t+2 a$ & + & - & - & + & - & {$[30]$} \\
\hline Amoimyrmex striatus & 22 & 0.35 & SC & Brazil & $20 m+2 s m$ & + & + & - & + & - & [11] \\
\hline Amoimyrmex silvestrii & 22 & & & Argentine & $20 \mathrm{~m}+2 \mathrm{sm}$ & & & & & & {$[36]$} \\
\hline
\end{tabular}


Table 5. Cont.

\begin{tabular}{|c|c|c|c|c|c|c|c|c|c|c|c|}
\hline Species Studied & $2 n(n)$ & $\begin{array}{c}\text { Genome } \\
\text { Size 1C } \\
\text { (pg) }\end{array}$ & Local/State & Country & Karyotype & \multicolumn{5}{|c|}{$\begin{array}{c}\text { Heterochromatic } \\
\text { Pattern } \\
\text { (C-Bands) }\end{array}$} & References \\
\hline Amoimyrmex bruchi & 22 & & & Argentine & $20 \mathrm{~m}+2 \mathrm{sm}$ & & & & & & [36] \\
\hline Apterostigma madidiense & $(23)$ & - & & Brazil & $14 \mathrm{~m}+20 \mathrm{sm}+10 \mathrm{st}+2 \mathrm{a}$ & & & & & & {$[37]$} \\
\hline Apterostigma madidiense & 24 & 0.74 & MG & Brazil & $24 \mathrm{~m}$ & + & + & - & - & - & This study \\
\hline Apterostigma mayri & 24 & - & & Panama & $24 \mathrm{~m}$ & + & - & - & - & - & [38] \\
\hline Apterostigma sp. & 20 & - & & Brazil & $6 m+12 s m+2 a$ & & & & & & [34] \\
\hline Apterostigma sp. & 24 & - & & Panama & $24 \mathrm{~m}$ & & & & & & [38] \\
\hline Apterostigma sp. & 32 & - & & French Guiana & $14 m+6 s m+10 s t+2 t$ & & & & & & {$[39]$} \\
\hline Apterostigma steigeri & 22 & - & & Brazil & $20 m+2 s m$ & & & & & & [37] \\
\hline Atta bisphaerica & 22 & - & MG & Brazil & $12 m+6 s m+4 a$ & + & + & - & - & - & {$[34,40]$} \\
\hline Atta colombica & $22(11)$ & 0.31 & & Panama & $12 m+6 s m+4 a$ & + & - & + & - & - & [38] \\
\hline Atta laevigata & 22 & 0.33 & MG & Brazil & $12 m+6 s m+4 a$ & + & + & - & - & - & {$[34,40]$} \\
\hline Atta sexdens & 22 & 0.33 & MG, RS & Brazil & $12 \mathrm{~m}+6 \mathrm{sm}+4 \mathrm{a}$ & + & + & - & - & - & {$[34,35,40]$} \\
\hline Atta sexdens & 22 & - & & French Guiana & $18 \mathrm{~m}+2 \mathrm{sm}+2 \mathrm{st}$ & + & + & - & - & - & {$[33]$} \\
\hline Cyphomyrmex cornutus & 22 & - & & French Guiana & $10 \mathrm{~m}+12 \mathrm{sm}$ & & & & & & [39] \\
\hline Cyphomyrmex costatus & 20 & - & & Panama & $20 \mathrm{~m}$ & + & - & - & - & - & {$[38]$} \\
\hline Cyphomyrmex rimosus & 32 & - & & Panama & $28 \mathrm{~m}+4 \mathrm{a}$ & & & & & & {$[38]$} \\
\hline Cyphomyrmex transversus & $24(12)$ & - & & French Guiana & $14 \mathrm{~m}+6 \mathrm{sm}+4 \mathrm{a}$ & & & & & & {$[33]$} \\
\hline Cyphomyrmex transversus & $42(21)$ & 0.50 & RJ & Brazil & $28 \mathrm{~m}+14 \mathrm{sm}$ & + & - & - & - & - & This study \\
\hline Mycetarotes carinatus & 14 & - & MG & Brazil & $8 m+6 s m$ & + & + & - & - & - & {$[42]$} \\
\hline Mycetarotes parallelus & 54 & 0.38 & MG & Brazil & $26 m+16 s m+6 a$ & + & + & - & + & - & [42] \\
\hline Mycetomoellerius fuscus & $18(9)$ & 0.47 & MG & Brazil & $16 \mathrm{~m}+2 \mathrm{sm}$ & + & + & - & - & - & {$[43]$} \\
\hline Mycetomoellerius holmgreni & $20(10)$ & 0.33 & $\begin{array}{c}\text { MG, SC, } \\
\text { RS }\end{array}$ & Brazil & $20 \mathrm{~m}$ & + & + & - & - & - & [7] \\
\hline Mycetomoellerius iheringi & $20(10)$ & 0.40 & $\mathrm{SC}$ & Brazil & $18 \mathrm{~m}+2 \mathrm{sm}$ & + & + & - & - & - & {$[44]$} \\
\hline
\end{tabular}


Table 5. Cont.

\begin{tabular}{|c|c|c|c|c|c|c|c|c|c|c|c|}
\hline \multirow{2}{*}{$\begin{array}{c}\text { Species Studied } \\
\text { Mycetomoellerius relictus }\end{array}$} & \multirow{2}{*}{$\begin{array}{l}\text { 2n (n) } \\
20(10)\end{array}$} & \multirow{2}{*}{$\begin{array}{c}\text { Genome } \\
\text { Size 1C } \\
\text { (pg) }\end{array}$} & \multirow{2}{*}{$\begin{array}{c}\text { Local/State } \\
\text { MG }\end{array}$} & \multirow{2}{*}{$\begin{array}{c}\text { Country } \\
\text { Brazil }\end{array}$} & \multirow{2}{*}{$\begin{array}{c}\text { Karyotype } \\
20 \mathrm{~m} \\
\end{array}$} & \multicolumn{5}{|c|}{$\begin{array}{l}\text { Heterochromatic } \\
\text { Pattern } \\
\text { (C-Bands) }\end{array}$} & \multirow{2}{*}{$\begin{array}{c}\text { References } \\
{[37]}\end{array}$} \\
\hline & & & & & & + & + & - & - & - & \\
\hline Mycetomoellerius sp. & 22 & - & MG & Brazil & $18 \mathrm{~m}+4 \mathrm{sm}$ & & & & & & [37] \\
\hline Mycetophylax conformis & $30(15)$ & 0.31 & RJ, SP & Brazil & $22 m+8 s m$ & + & + & - & + & - & [10] \\
\hline Mycetophylax morschi & $30(15)$ & 0.34 & RJ, RS, SC & Brazil & $18 m+6 s m+2 a$ & + & - & - & - & - & [10] \\
\hline Mycetophylax morschi & $28(14)$ & - & BA & Brazil & $18 \mathrm{~m}+10 \mathrm{sm}$ & + & - & - & - & - & [17] \\
\hline Mycetophylax simplex & $36(18)$ & 0.41 & SC, PR, SP & Brazil & $20 m+16 s m$ & + & + & - & + & - & [10] \\
\hline Mycocepurus goeldii & 8 & - & MG & Brazil & $8 \mathrm{~m}$ & + & + & - & - & - & [45] \\
\hline Mycocepurus goeldii & $8(4)$ & 0.42 & SC & Brazil & $4 m+4 s m$ & & & & & & This study \\
\hline Mycocepurus sp. & 8 & - & & Panama & $4 \mathrm{~m}$ & & & & & & {$[38]$} \\
\hline Myrmicocrypta sp. & 30 & - & & French Guiana & $22 m+2 s m+6 a$ & & & & & & {$[33]$} \\
\hline Sericomyrmex amabilis & 50 & 0.45 & & Panama & $50 \mathrm{~m}$ & + & + & - & - & - & [38] \\
\hline Sericomyrmex sp. & $50(25)$ & - & MG & Brazil & $44 \mathrm{~m}+6 \mathrm{sm}$ & & & & & & [37] \\
\hline Serycomyrmex parvulus & $50(25)$ & 0.42 & MG & Brazil & $30 m+14 s m+6 s t$ & + & + & - & - & - & This study \\
\hline Trachymyrmex septentrionalis & $20(10)$ & 0.25 & & Panama & $20 \mathrm{~m}$ & & & & & & [38] \\
\hline Trachymyrmex sp.1 & $12(6)$ & - & & Panama & $12 \mathrm{~m}$ & + & - & + & - & - & [38] \\
\hline Trachymyrmex sp.2 & 18 & - & & Panama & $18 \mathrm{~m}$ & & & & & & [38] \\
\hline
\end{tabular}




\section{Discussion}

\subsection{Chromosome Number, Mode, and Tempo of Karyotype Change}

Genes are scattered across the genome, which in eukaryotes, are organized and distributed in a different number of DNA molecules with differing extents. These molecules are complexed with proteins and comprise the chromosomes that are ultimately the units of inheritance. Ants exhibit an astonishing variation in chromosome number and structure (e.g., [17]) that, it has been postulated, take part in ant speciation and diversification. They show a haplodiploid sex-determination system, as in all Hymenoptera, with haploid chromosome numbers ranging from $n=1$ to $n=60$, and include diverse karyotypes comprising various types of chromosomes (see [2]. Of the subfamilies of Formicidae, Myrmicinae is the subfamily with the greatest number of studied species and taxa from a cytogenetic perspective [3]. Here, we have added six new chromosome counts to the remarkable fungus-farming ants that live in symbiosis with Basidiomycete fungi inside their subterranean nests across the Americas.

According to our results, approximately 58 taxa in 13 out of 18 genera have been studied. Considering only the published data, the diploid karyotype of fungus-farming ants varies from 4 haploid chromosomes in Mycocepurus goeldii (Forel, 1893) up to 27 haploid chromosomes in Mycetarotes parallelus (Roger, 1863). The diploid chromosome numbers are, in general, variable in fungus-farming ants, as is the genome size (Figure 6). However, the variability in chromosome number and genome size is highly unequally distributed among fungus-farming ant genera, with most of the variability concentrated in the early diverged lineages/genera, such as in Mycetophylax and Mycetarotes. In contrast, other lineages have much more stable chromosome numbers, such as $n=11$ in the leafcutting ants from the genera Atta and Amoimyrmex, as well as $n=19$ in the leafcutting ants from the genus Acromyrmex.

The chromosome number $n=10$ or $n=11$ exhibits a notable long-term evolutionary stasis across fungus-farming ant lineages (see Figure 6), also refereed as intermediate evolutionary statis. Karyotypes with $n=11$ chromosomes were identified in the Apterostigma clade and in the Cyphomyrmex clade dated to $\sim 45 \mathrm{Mya}$ and $\sim 25 \mathrm{Mya}$, respectively. The same chromosome number was identified in Amoimyrmex and Atta clades that are much more recent, estimated to $\sim 4.7$ Mya and $\sim 13.7$ Mya, respectively. However, the $n=10$ chromosomes that were identified in the Cyphomyrmex clade ("costatus group"), the Mycetomollerius clade, and the Trachymyrmex clade are estimated at approximately $\sim 19$ Mya, $\sim 24.8 \mathrm{Mya}$, and $\sim 16.8 \mathrm{Mya}$, respectively (Figure 6 and Figure S1). This pattern of chromosome number variation and distribution was also observed in other Hymenoptera groups, such as stingless bees [46] and parasitoid wasps [47].

Considering the phylogenetic relationship of fungus-farming species, the general slight variation in genome size across lineages, with few exceptions, shows that a low chromosome number between 10 to 12 haploid chromosome and genome size near to $0.38 \mathrm{pg}$ (see Figure 6), should be plesiomorphic features. Indeed, a low ancestral GS of $\sim 0.37-0.38$ has been estimated and proposed for Formicidae [16] and the chromosome number between 10 to 12 of metacentric/submetacentric chromosomes spreads widely across fungus-farming ant lineages. Thus, considering the tight GS in fungus-farming ants, the observed differences in chromosome number across fungus-farming ant genera suggests that changes in chromosome numbers evolve gradually over time, via multiple rearrangement events that culminate in different races due to the fixation of a single or a few chromosomal changes, followed by the extinction of intermediate karyotypes (see $[10,17,48-50])$. Therefore, karyotypes $n \leq 15$ and GSs of approximately 0.38 pg should be frequent in fungus-farming ants, whereas karyotypes $n>15$ and GS $>0.38 \mathrm{pg}$ are exceptions and are restricted to only some fungus-farming ant lineages. The accumulation of repetitive DNA appears to be mainly responsible for genome size expansions, instead of genome duplication see [16]. These increases in repetitive DNA may promote changes in chromosome number since the heterochromatic regions are hotspots for chromosome 
breakpoints [51]. In fact, given its nature and critical function, heterochromatin is considered to be a rapidly evolving genomic component that can promote species divergence [52]. As an example, there is a small GS variation among leafcutting ants (Atta, Acromyrmex, and Amoimyrmex), and the chromosome number is indeed consistent within each genus (Figure 6). However, in contrast with Atta and Amoimyrmex, the karyotypes of Acromyrmex are $n=19(n>15)$ and show a great distribution of heterochromatic regions, as revealed by the C-banding [32]. Similarly, Mycetophylax simplex $n=18(n>15)$ also shows a much higher number of $\mathrm{C}$-banded heterochromatic regions compared with the sister species studied cytogenetically [10].

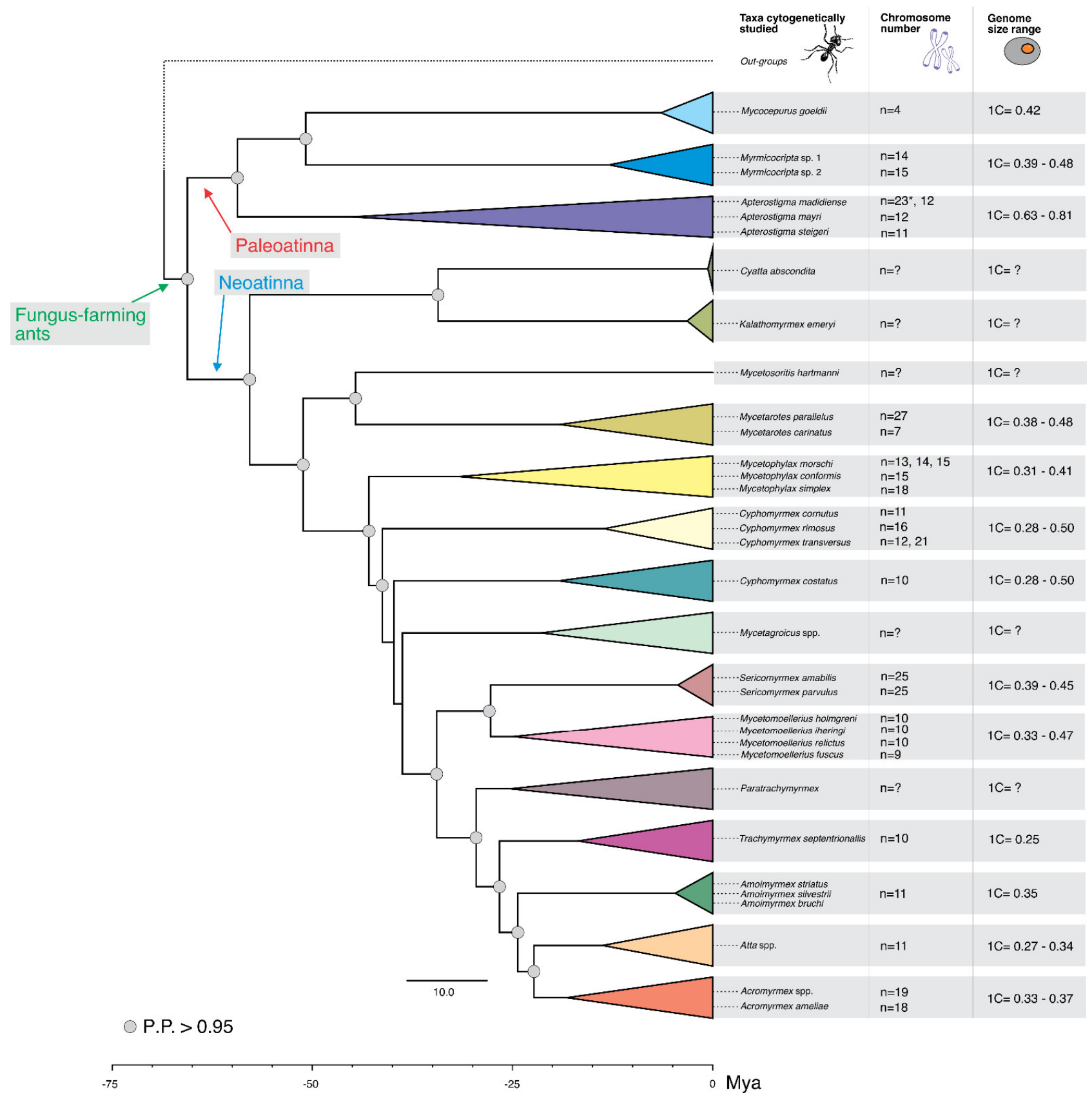

Figure 6. Cytogenetic information regarding fungus-farming ants mapped on the time-calibrated phylogenetic tree of subtribe Attina (Myrmicinae) aiming genera. Available chromosome numbers and genome size for certain taxa are given (data refer to Table 5). Asterisk: previously published chromosome report that differs from the number published in the present study. 
Chromosomal rearrangements should thus promote species isolation and lineage diversification because of the reduced recombination between heterokaryotypes [53]. The rate at which chromosomes change may be related to the amount and distribution of heterochromatin. The repetitive DNA comprising heterochromatin may promote rearrangements by relaxing the putative selective pressure on gene synteny and linkage groups. Indeed, the poorly heterochromatin-rich karyotypes show a chromosome number of $n \leq 15$, as observed in Mycetomollerius and Cyphomyrmex (see Table 1 and Figure 6). Most fungus-farming ants studied to date show heterochromatin restricted to the centromeric region $[7,10,11,32,37,43]$. Further studies covering more data and combining genomic information may shed more light on the above-proposed hypothesis of chromosome number variation and diversity.

Karyotype evolution in ants is commonly explained in terms of the "minimum interaction theory-MIT" proposed by Imai et al. [4,5] as a general mechanism to explain the chromosomal change in ants. Following the MIT, over evolutionary time, the number of chromosomes in the ant karyotypes will increase, whereas chromosome size will decrease. Such a pattern minimizes the threat of deleterious rearrangements due to the interaction of chromosomes inside the nucleus. The predicted increase in chromosome number is due to centric fission that represents the main chromosome rearrangements in ants, followed by chromatin addition (mainly heterochromatin) or pericentromeric inversions (see [5]). Although the minimum interaction theory does not disregard fusions, such a rearrangement is considered rare and fixed or positively selected when it brings about short-term advantages [5,48].

Accordingly, in the course of evolution, chromosomes will increase in number and reduce in size. Taking the cytogenetic data available to date, phylogenetic relationships, and the time of divergence studied here, a higher chromosome number would be expected in ancient lineages than in recent lineages. The higher chromosome number known is observed in Mycetarotes parallelus $(n=27)$, but unexpectedly low karyotypes, such as $n<15$, are found in the anciently divergent clade called Paleoattina, which comprises Mycocepurus, Myrmicocripta, and Aptersotigma (Figure 6). Furthermore, higher counts, such as $n=25$ or $n=19(n>15)$, are scattered in the clade comprising recently divergent lineages such as Sericomyrmex and Acromyrmex. The cytogenetic data plotted in the phylogeny reveals that the karyotype $n=10$, or a number close to that ( $n=11$ and 12), is the most common karyotype across fungus-farming ant genera, showing that the karyotype of the lineages is around the average or the most frequent number. Thus, we do not discard that the minimum interaction theory may be a driving force behind karyotype evolution in ants; however, it seems that other forces may regulate chromosome number by imposing a general common karyotype number, such as $n<15$, in fungus-farming ants. A few exceptions where the karyotype reaches higher chromosome numbers appear to overcome the barrier likely caused by the expansion of repetitive genomic elements [16]. This is in agreement with the heterochromatic pattern observed in fungus-farming ants. Karyotypes with a haploid number over 15 chromosomes show a higher number of heterochromaticpositive bands. This also matches with the MIT, which presumes that after centric fission occurs, the duplication of repetitive regions at the extreme of the newly produced telocentric/acrocentric chromosomes creates stabilization [5]. Such processes will increase the number of heterochromatic segments and will promote the increase in GS, not as abruptly as polyploidization, but as we observed across the estimated GS values $[16,54]$ (see Figure 6).

\subsection{Is There an Upper Limit to the Chromosome Number of Fungus-Farming Ants?}

The answer to that question is intriguing and complex. The chromosome number ultimately means how many DNA molecules the genome of any organism comprises. A fundamental property of the genome is how the genes are distributed along and among the chromosomes, which has a strong influence on gene function and regulation. Ants exhibit an astonishing variation in chromosome numbers, from a species that starts with 
a minimum of $n=1$ in the Australian bulldog ant Myrmecia croslandi [55], only possible due to the haplodiploid sex determination, up to as much as $n=60$ in the giant neotropical ant Dinoponera lucida [56]. In contrast, such a huge diversity is not observed across known genome size values, which vary from 0.18 to $0.81 \mathrm{pg}$ [16]. Chromosome numbers vary on a scale of 60 times (from 1 to 60 ), whereas genome size is below 4.5 times. Presumably, there is an evolutionary constraint preventing genome expansion up to a certain limit, and similarly, there would be an upper limit to chromosome number. Thus, the answer to our initial question is: yes!

The maximum number of chromosomes known thus far for fungus-farming ants is $n=27$ for one record and $n=25$ for another (see Table 1 ). The scarcity of karyotypes with large chromosome numbers and the high frequency of karyotypes with $n<15$ suggests a potential constraint in chromosome number. Here, based on genome size data in which great departures from the mean GS are rare, we propose that there is an upper limit for chromosome number. If not, we would observe karyotypes above $n=15$ more often and higher GS estimates. According to the MIT, the higher the chromosome number is, the smaller the chromosomes are, and this is a fact observed in our karyomorphometric data. In addition, according to the MIT, centric fission events producing unstable telocentric/acrocentric chromosomes promote heterochromatin expansion, resulting in an increase in DNA [5]. However, the limited range of GSs observed in fungus-farming ants is the first instance of evidence for an upper limit in chromosome numbers. Fusion events counterbalance centric fissions due to the loss of DNA see $[4,5]$. Furthermore, the progressive reduction in the size of the chromosomes as stated by the MIT may increase the risk of mis-segregation of chromosomes during cell division and dysploidy [57]. For instance, the species Dinoponera lucida, which bears the highest number of chromosomes in Formicidae, shows huge variation in chromosome number across populations [56].

Several hypotheses have been invoked and tested to explain the tight genome size of ants: the developmental strategy (holometabolous) and metabolic rate have been suggested as constraints against genome size expansion, as well as the eusociality $[58,59]$. Similarly, such mechanisms may regulate the upper limit of chromosomes in ants. A GS above $0.70 \mathrm{pg}$ is rare, and is suggested to eventually occur from whole-genome duplication (double the mean GS of $0.38 \mathrm{pg}$ ). Most ants show a GS of between 0.25 and $0.50 \mathrm{pg}$, and such a range is suggested to be the result of transposition element (TE) activity and repetitive DNA expansion rather than whole-genome duplication [16]. It is a fact that the ant genome sizes estimated so far are always below $1 \mathrm{pg}$. There are still open questions that need to be addressed, including: which genetic mechanism is triggered? And which evolutionary process may cause the loss and gain of DNA in ants maintaining such a small genome? Some evidence suggests that DNA loss is likely to be favored compared with gain by selection to minimize genetic instability, or phenotypic effects due to the required changes in the nucleus and cell size [60]. Furthermore, there are biochemical and energy costs associated with maintaining expanded genomes, as well the cellular machinery involved in chromosome organization and splitting during cell division [61]. This is in agreement with the minimum interaction theory and the implicated processes (centric fissions) responsible for the chromosomal change (see [5]); however, we hypothesize that there is a balance between fission and fusion events. The constricted genome size, the expansion constraints, the TE activity promoting insertions, and duplication may trigger chromosome rearrangements within an upper limit number of DNA molecules. Thus, there is an upper limit in chromosome number, but not for karyotype shifting (inversions, translocations, etc.), which may explain the dynamic and diverse karyotype of ants. Further genomic data and chromosome-level assembly studies may shed light on ant chromosome biology. It is a fact that there are, in nature, karyotypes bearing over hundreds of chromosomes, such as in the butterfly Polyommatus (Plebicula) atlanticus ( $n$ = circa 223) [62]. However, it seems they are the exceptions, rather than the rule. 


\subsection{Chromosome Counts: How Far We Get and What Is Still Needed}

In this paper, we evaluated the currently available cytogenetic information on fungus-farming ants, a distinguishable group of neotropical fauna. From the data studied to date, it is possible to make some assumptions regarding chromosome numbers, karyotype evolution, and genome size variation. Among all the ant species, the highest amount of cytogenetic data available is on the Myrmicinae, specifically fungus-farming ants. However, considering the diversity of ants and their ecological importance, we have only superficially touched on a much deeper compendium of knowledge when we consider that less than five percent of ants has been studied cytogenetically thus far [3]. In our opinion, the chromosome number is still essential, and we need to continue counting. Furthermore, we need to couple cytogenetic information with molecular and genomic data. Considering the generally tight genome size of ants and the karyotype diversity, the interplay between fission-fusion and other structural rearrangements, such as duplication and inversions, may govern karyotype evolution in fungus-farming ants. We can also extrapolate this to all ants. It is likely that changes in chromosome number caused by fission, fusion, inversions, and duplications may have contributed to ant diversification $[2,3]$. Thus, cytogenetic studies can definitely contribute to ant systematics, taxonomy, and evolutionary biology.

\section{Conclusions}

In this paper, we reported two chromosome counts that differ from previously published chromosome reports for the same species. This raises issues that need to be addressed in an effort to gain cytogenetic knowledge and application: first, an unidentified species may be tagged with cytochrome oxidase I ( $\operatorname{cox} 1$ or COI) sequences for further identification (see [63]); second, different counts may suggest independent lineages and new potential species; third, chromosome counting needs protocol scrutiny with regard to the number of cell/metaphases assessed in colonies/individuals to avoid flawed reports. Bearing in mind the well-known case of the human karyotype where, for more than 30 years, it was thought to be 48 diploid chromosomes instead of 46 (see [64]), in the chromosome counting of ants, and Hymenoptera in general, evaluating a minimum of individuals and cells should be considered. Other issues that require attention are the observance of source tissue (male or female), the number of cells/individuals per colony, and the number of colonies. The haplodiploid sex determination prevents chromosome paring from male samples, which could result in a misinterpretation of numbers. Increasing the number of cells/individuals/colonies decreases the propensity of errors. Due to sampling difficulties or bias, highlighting the concerns in reporting such data is necessary for future studies. In conclusion, the phylogenetic relationship of fungus-farming ants and the cytogenetic data presented here suggest that a low chromosome number between 10 to 12 haploid chromosome and genome size near to $0.38 \mathrm{pg}$ should be plesiomorphic features. The karyotype variation observed today is a result of an interplay among distinct chromosomal rearrangements and likely lineage-specific.

Supplementary Materials: The following are available online at https:/ /www.mdpi.com/article/ 10.3390/insects12121084/s1, Table S1: Models of molecular evolution by genes and codons implemented in the Bayesian analyses to infer the molecular phylogeny of fungus-growing ants. Figure S1: Chronogram of fungus-farming ants estimated by fossilized birth-death (FDB) model based on five molecular markers (see Materials and Methods).

Author Contributions: M.P.C. and D.C.C. conceived and designed the experiments; M.P.C. and D.C.C. performed the experiments; M.P.C. and D.C.C. analyzed the data; M.P.C. and D.C.C. contributed materials/analysis tools; M.P.C. and D.C.C. wrote the manuscript. All authors have read and agreed to the published version of the manuscript.

Funding: D.C.C. wishes to thank the Fundação de Amparo à Pesquisa do Estado de Minas Gerais-FAPEMIG (PPM0199-18) for providing financial support. The authors also thank Conselho Nacional de Desenvolvimento Científico e Tecnológico-CNPq for the research fellowships: D.C.C.-312900/2020-1 
and M.P.C.-309579/2018-0. Funding agencies had no role in the design, collection, analysis or interpretations of the data in the present study.

Institutional Review Board Statement: Not applicable.

Data Availability Statement: All data are referenced and available in the manuscript.

Acknowledgments: We are grateful to the many people who made this work possible. We thank all of our colleagues at the Lab and Research Group of Genetics and Evolution of Ants (GEF-UFOP) for their help with data and sampling over the years. We also thank Mariana Neves Moura for the fieldwork help and Ana Carolina Coelho dos Reis for the initial assistance in statistical work. We thank Tiago Degrandi, Marina Cunha, the editor and two reviewers for their helpful comments on previous versions of the manuscript.

Conflicts of Interest: The authors declare no conflict of interest. The funders had no role in the design of the study; in the collection, analyses, or interpretation of data; in the writing of the manuscript, or in the decision to publish the results.

\section{References}

1. King, M. Species Evolution: The Role of Chromosome Change; Cambridge University Press: Cambridge, UK, 1993.

2. Lorite, P.; Palomeque, T. Karyotype evolution in ants (Hymenoptera: Formicidae), with a review of the known ant chromosome numbers. Myrmecol. News 2010, 13, 89-102.

3. Cardoso, D.C.; Santos, H.G.; Cristiano, M.P. The Ant Chromosome database-(ACdb): An online resource for ant (Hymenoptera: Formicidae) chromosome researchers. Myrmecol. News 2018, 27, 87-91.

4. Imai, H.T.; Taylor, R.W.; Crozier, R.H. Modes of spontaneous chromosomal mutation and karyotype evolution in ants with reference to the minimum interaction hypothesis. Jpn. J. Genet. 1988, 63, 159-185. [CrossRef] [PubMed]

5. Imai, H.T.; Taylor, R.W.; Crozier, R.H. Experimental bases for the minimum interaction theory. I. Chromosome evolution in ants of the Myrmecia pilosula species complex (Hymenoptera: Formicidae: Myrmeciinae). Jpn. J. Genet. 1994, 69, 137-182. [CrossRef]

6. Speicher, M.R.; Carter, N.P. The new cytogenetics: Blurring the boundaries with molecular biology. Nat. Rev. Genet. 2005, 6, 782-792. [CrossRef]

7. Cardoso, D.C.; Heinze, J.; Moura, M.N.; Cristiano, M.P. Chromosomal variation among populations of a fungus-farming ant: Implications for karyotype evolution and potential restriction to gene flow. BMC Evol. Biol. 2018, 18, 146. [CrossRef]

8. Micolino, R.; Cristiano, M.P.; Cardoso, D.C. Population-Based Cytogenetic Banding Analysis and Phylogenetic Relationships of the Neotropical Fungus-Farming Ant Trachymyrmex holmgreni Wheeler, 1925. Cytogenet. Genome Res. 2019, 159, $151-161$. [CrossRef]

9. Guerra, M. Cytotaxonomy: The end of childhood. Plant Biosyst. 2012, 146, 703-710.

10. Cardoso, D.C.; Pompolo, S.G.; Cristiano, M.P.; Tavares, M.G. The role of fusion in ant chromosome evolution: Insights from cytogenetic analysis using a molecular phylogenetic approach in the genus Mycetophylax. PLoS ONE 2014, 9, e87473.

11. Cristiano, M.P.; Cardoso, D.C.; Fernandes-Salomão, T.M. Cytogenetic and molecular analyses reveal a divergence between Acromyrmex striatus (Roger, 1863) and other congeneric species: Taxonomic implications. PLoS ONE 2013, 8, e59784. [CrossRef]

12. Schultz, T.R.; Brady, S.G. Major evolutionary transitions in ant agriculture. Proc. Natl. Acad. Sci. USA 2008, 105, 5435-5440. [CrossRef]

13. Branstetter, M.G.; Ješovnik, A.; Sosa-Calvo, J.; Lloyd, M.W.; Faircloth, B.C.; Brady, S.G.; Schultz, T.R. Dry habitats were crucibles of domestication in the evolution of agriculture in ants. Proc. Biol. Sci. 2017, 284, 20170095. [CrossRef]

14. Solomon, S.E.; Rabeling, C.; Sosa-Calvo, J.; Lopes, C.T.; Rodrigues, A.; Vasconcelos, H.L.; Bacci, M.; Mueller, U.G.; Schultz, T.R. The molecular phylogenetics of Trachymyrmex Forel ants and their fungal cultivars provide insights into the origin and coevolutionary history of 'higher-attine' ant agriculture. Syst. Entomol. 2019, 44, 939-956. [CrossRef]

15. Cristiano, M.P.; Cardoso, D.C.; Sandoval-Gómez, V.E.; Simões-Gomes, F.C. Amoimyrmex Cristiano, Cardoso \& Sandoval, gen. nov. (Hymenoptera: Formicidae): A new genus of leaf-cutting ants revealed by multilocus molecular phylogenetic and morphological analyses. Austral Entomol. 2020, 59, 643-676.

16. Moura, M.N.; Cardoso, D.C.; Cristiano, M.P. The tight genome size of ants: Diversity and evolution under ancestral state reconstruction and base composition. Zool. J. Linn. Soc. 2021, 193, 124-144. [CrossRef]

17. Micolino, R.; Cristiano, M.P.; Travenzoli, N.M.; Lopes, D.M.; Cardoso, D.C. Chromosomal dynamics in space and time: Evolutionary history of Mycetophylax ants across past climatic changes in the Brazilian Atlantic coast. Sci. Rep. 2019, 9, 18800. [CrossRef]

18. Cardoso, D.C.; Cristiano, M.P.; Tavares, M.G. Methodological remarks on rearing basal Attini ants in the laboratory for biological and evolutionary studies: Overview of the genus Mycetophylax. Insectes Sociaux 2011, 58, 427-430. [CrossRef]

19. Cardoso, D.C.; Pereira, T.T.P.; Cordeiro, A.L.; Cristiano, M.P. Cytogenetic data on the agro-predatory ant Megalomyrmex incisus Smith, 1947 and its host, Mycetophylax conformis (Mayr, 1884) (Hymenoptera, Formicidae). Comp. Cytogenet. 2017, 11, 45-53. [CrossRef] 
20. Levan, A.; Fredga, K.; Sandberg, A. Nomenclature for centromeric position on chromosomes. Hereditas 1964, 52, $201-220$. [CrossRef]

21. Cristiano, M.P.; Pereira, T.T.P.; Simões, L.P.; Sandoval-Gómez, V.E.; Cardoso, D.C. Reassessing the Chromosome Number and Morphology of the Turtle Ant Cephalotes pusillus (Klug, 1824) Using Karyomorphometrical Analysis and Observations of New Nesting Behavior. Insects 2017, 8, 114. [CrossRef]

22. Sumner, A.T. A simple technique for demonstrating centromeric heterochromatin. Exp. Cell Res. 1972, 75, 304-306. [CrossRef]

23. Bouckaert, R.; Vaughan, T.G.; Barido-Sottani, J.; Duchêne, S.; Fourment, M.; Gavryushkina, A.; Heled, J.; Jones, G.; Kühnert, D.; De Maio, N.; et al. BEAST 2.5: An advanced software platform for Bayesian evolutionary analysis. PLoS Comput. Biol. 2018, 15, e1006650. [CrossRef] [PubMed]

24. Heath, T.A.; Huelsenbeck, J.P.; Stadler, T. The fossilized birth-death process for coherent calibration of divergence-time estimates. Proc. Natl. Acad. Sci. USA 2014, 111, 2957-2966. [CrossRef] [PubMed]

25. Lanfear, R.; Frandsen, P.; Wright, A.; Senfeld, T.; Calcott, B. PartitionFinder 2: New methods for selecting partitioned models of evolution for molecular and morphological phylogenetic analyses. Mol. Biol. Evol. 2016, 34, 772-773. [CrossRef]

26. Rambaut, A. FigTree v1.4.3. 2009. Available online: http:/ / tree.bio.ed.ac.uk/software/figtree (accessed on 10 January 2020).

27. Rambaut, A.; Drummond, A.; Xie, D.; Baele, G.; Suchard, M. Posterior summarization in Bayesian phylogenetics using Tracer 1.7. Syst. Biol. 2018, 67, 901-904. [CrossRef]

28. Goñi, B.; De Zolessi, L.C.; Imai, H.T. Karyotypes of thirteen ant species from Uruguay (Hymenoptera, Formicidae). Caryologia 1983, 36, 363-371. [CrossRef]

29. Castro, C.P.M.; Cardoso, D.C.; Micolino, R.; Cristiano, M.P. Comparative FISH-mapping of TTAGG telomeric sequences to the chromosomes of leafcutter ants (Formicidae, Myrmicinae): Is the insect canonical sequence conserved? Comp. Cytogenet. 2020, 14, 369-385. [CrossRef]

30. Barros, L.A.C.; Aguiar, H.J.A.C.; Teixeira, G.C.; Souza, D.J.; Delabie, J.H.C.; Mariano, C.S.F. Cytogenetic studies on the social parasite Acromyrmex ameliae (Formicidae: Myrmicinae: Attini) and its hosts reveal chromosome fusion in Acromyrmex. Zool. Anz. 2021, 293, 273-281. [CrossRef]

31. Teixeira, G.A.; Barros, L.A.C.; Aguiar, H.J.A.C.; Pompolo, S.G. Comparative physical mapping of $18 \mathrm{~S}$ rDNA in the karyotypes of six leafcutter ant species of the genera Atta and Acromyrmex (Formicidae: Myrmicinae). Genetica 2017, 145, 351-357. [CrossRef]

32. Barros, L.A.C.; de Aguiar, H.; Mariano, C.D.F.; Andrade-Souza, V.; Costa, M.A.; Delabie, J.H.C.; Pompolo, S.D. Cytogenetic data on six leafcutter ants of the genus Acromyrmex Mayr, 1865 (Hymenoptera, Formicidae, Myrmicinae): Insights into chromosome evolution and taxonomic implications. Comp. Cytogenet. 2016, 10, 229-243. [CrossRef]

33. Aguiar, H.J.A.C.; Barros, L.A.C.; Silveira, L.I.; Petitclerc, F.; Etienne, S.; Orivel, J. Cytogenetic data for sixteen ant species from North-eastern Amazonia with phylogenetic insights into three subfamilies. Comp. Cytogenet. 2020, 14, 43-60. [CrossRef]

34. Fadini, M.A.M.; Pompolo, S.G. Cytogenetics of some ant species of the tribe Attini (Hymenoptera, Formicidae) from the region of Viçosa, MG. Rev. Bras. Genet. 1996, 19, 53-55.

35. Santos-Colares, M.C.; Viégas, J.; Roth, M.G.M.; Loeck, A.E. Preparation of mitotic chromosomes of leaf-cutting ants from the genera Atta and Acromyrmex. Braz. J. Genet. 1997, 20, 25-27. [CrossRef]

36. Micolino, R.; Baldez, B.C.L.; Sánchez-Restrepo, A.F.; Calcaterra, L.; Cristiano, M.P.; Cardoso, D.C. Karyotype structure and cytogenetic markers of Amoimyrmex bruchi and Amoimyrmex silvestrii: Contribution to understanding leaf-cutting ant relationships. Genome 2021, in press. [CrossRef]

37. Barros, L.A.C.; Mariano, C.S.F.; Pompolo, S.G. Cytogenetic studies of five taxa of the tribe Attini (Formicidae: Myrmicinae). Caryologia 2013, 66, 59-64. [CrossRef]

38. Murakami, T.; Fujiwara, A.; Yoshida, M.C. Cytogenetics of ten ant species of the tribe Attini (Hymenoptera, Formicidae) in Barro Colorado Island, Panama. Chromosome Sci. 1998, 2, 135-139.

39. Mariano, C.S.F.; Santos, I.D.; Groc, S.; Leroy, C.; Malé, P.J.; Ruiz-González, M.X.; Cerdan, P.; Dejean, A.; Delabie, J.H.C. The karyotypes of Gigantiops destructor (Fabricius) and other ants from French Guiana (Formicidae). Ann. Société Entomol. Fr. 2011, 47, 140-146. [CrossRef]

40. Barros, L.A.C.; Teixeira, G.A.; Aguiar, H.J.A.C.; Mariano, C.S.F.; Delabie, J.H.C.; Pompolo, S.G. Banding Patterns of Three Leafcutter Ant Species of the Genus Atta (Formicidae: Myrmicinae) and Chromosomal Inferences. Fla. Entomol. 2014, 97, 1694-1701. [CrossRef]

41. Barros, L.A.; Aguiar, H.J.; Teixeira, G.A.; Mariano, C.S.; Teixeira, M.C.; Delabie, J.H.; Pompolo, S.d. Cytogenetic data on the threatened leafcutter ant Atta robusta Borgmeier, 1939 (Formicidae: Myrmicinae: Attini). Comptes Rendus Biol. 2015, 338, 660-665. [CrossRef]

42. Barros, L.A.C.; Mariano, C.S.F.; Pompolo, S.G.; Delabie, J.H.C. Citogenética de Attini. In Formigas-Cortadeiras: Da Bioecologia ao Manejo; Della-Lucia, T.M.C., Ed.; UFV: Viçosa, Brazil, 2011.

43. Barros, L.A.C.; Aguiar, H.J.A.C.; Mariano, C.S.F.; Delabie, J.H.C.; Pompolo, S.G. Cytogenetic characterization of the ant Trachymyrmex fuscus Emery, 1934 (Formicidae: Myrmicinae: Attini) with the description of a chromosomal polymorphism. Ann. Soc. Entomol. Fr. 2013, 49, 367-373. [CrossRef]

44. Micolino, R.; Cristiano, M.P.; Cardoso, D.C. Karyotype and putative chromosomal inversion suggested by integration of cytogenetic and molecular data of the fungus-farming ant Mycetomoellerius iheringi Emery, 1888. Comp. Cytogenet. 2020, 14, 197-210. [CrossRef] [PubMed] 
45. Barros, L.A.C.; Aguiar, H.J.A.C.; Mariano, C.S.F.; Delabie, J.H.C.; Pompolo, S.G. Cytogenetic characterization of the lower-Attini Mycocepurus goeldii (Formicidae: Myrmicinae: Attini). Sociobiology 2010, 56, 57-66.

46. Travenzoli, N.M.; Cardoso, D.C.; Werneck, H.d.A.; Fernandes-Salomão, T.M.; Tavares, M.G.; Lopes, D.M. The evolution of haploid chromosome numbers in Meliponini. PLoS ONE 2019, 14, e0224463. [CrossRef] [PubMed]

47. Gokhman, V.E.; Kuhn, K.L.; Woolley, J.B.; Hopper, K.R. Variation in genome size and karyotype among closely related aphid parasitoids (Hymenoptera, Aphelinidae). Comp. Cytogenet. 2017, 11, 97-117. [CrossRef] [PubMed]

48. Imai, H.T.; Satta, Y.; Takahata, N. Integrative study on chromosome evolution of mammals, ants and wasps based on the minimum interaction theory. J. Theor. Biol. 2001, 210, 475-497. [CrossRef] [PubMed]

49. Rieseberg, L.H. Chromosomal rearrangements and speciation. Trends Ecol. Evol. 2001, 16, 351-358. [CrossRef]

50. Lukhtanov, V.A.; Dincă, V.; Talavera, G.; Vila, R. Unprecedented within-species chromosome number cline in the Wood White butterfly Leptidea sinapis and its significance for karyotype evolution and speciation. BMC Evol. Biol. 2011, 11, 109. [CrossRef] [PubMed]

51. Grewal, S.I.; Jia, S. Heterochromatin revisited. Nat. Rev. Genet. 2007, 8, 35-46. [CrossRef]

52. Hughes, S.E.; Hawley, R.S. Heterochromatin: A Rapidly Evolving Species Barrier. PLoS Biol. 2009, 7, e1000233. [CrossRef]

53. Navarro, A.; Barton, N.H. Chromosomal speciation and molecular divergence-accelerated evolution in rearranged chromosomes. Science 2003, 11, 321-324. [CrossRef]

54. Tsutsui, N.D.; Suarez, A.V.; Spagna, J.C.; Johnston, J.S. The evolution of genome size in ants. BMC Evol. Biol. 2008, 8, 64-72. [CrossRef]

55. Crosland, M.W.; Crozier, R.H. Myrmecia pilosula, an ant with only one pair of chromosomes. Science 1986, 231, 1278. [CrossRef]

56. Mariano, C.D.S.F.; Pompolo, S.D.G.; Barros, L.A.C.; Mariano-Neto, E.; Campiolo, S.; Delabie, J.H.C.A. A biogeographical study of the threatened ant Dinoponera lucida Emery (Hymenoptera: Formicidae: Ponerinae) using a cytogenetic approach. Insect Conserv. Divers. 2008, 1, 161-168. [CrossRef]

57. Schubert, I. Alteration of chromosome numbers by generation of minichromosomes-Is there a lower limit of chromosome size for stable segregation? Cytogenet. Cell Genet. 2001, 93, 175-181. [CrossRef]

58. Ardila-Garcia, A.M.; Umphrey, G.J.; Gregory, T.R. An expansion of the genome size dataset for the insect order Hymenoptera, with a first test of parasitism and eusociality as possible constraints. Insect Mol. Biol. 2010, 19, 337-346. [CrossRef]

59. Gregory, T.R. Genome size evolution in animals. In The Evolution of the Genome; Gregory, T.R., Ed.; Elsevier: San Diego, CA, USA, 2005; pp. 3-87.

60. Kraaijeveld, K. Genome Size and Species Diversification. Evol. Biol. 2010, 37, 227-233. [CrossRef]

61. Hessen, D.O.; Jeyasingh, P.D.; Neiman, M.; Weider, L.J. Genome streamlining and the elemental costs of growth. Trends Ecol. Evol. 2010, 25, 75-80. [CrossRef]

62. Lukhtanov, V.A. The blue butterfly Polyommatus (Plebicula) atlanticus (Lepidoptera, Lycaenidae) holds the record of the highest number of chromosomes in the non-polyploid eukaryotic organisms. Comp. Cytogenet. 2015, 9, 683-690. [CrossRef]

63. Lukhtanov, V.A.; Pashenkova, Y. Linking karyotypes with DNA barcodes: Proposal for a new standard in chromosomal analysis with an example based on the study of Neotropical Nymphalidae (Lepidoptera). Comp. Cytogenet. 2019, 13, 435-449. [CrossRef]

64. Gartler, S.M. The chromosome number in humans: A brief history. Nat. Rev. Genet. 2006, 7, 655-660. [CrossRef] 